\title{
Line simplification with restricted orientations
}

\section{Report}

Author(s):

Neyer, Gabriele

Publication date:

1998

Permanent link:

https://doi.org/10.3929/ethz-a-006653098

Rights / license:

In Copyright - Non-Commercial Use Permitted

Originally published in:

Technical Report / ETH Zurich, Department of Computer Science 311 


\title{
Line Simplification with Restricted Orientations ${ }^{\dagger}$
}

\author{
G. Neyer
}

May 11, 1999

\begin{abstract}
We study the $\mathcal{C}$-oriented line simplification problem: Given a polygonal chain $P$ represented by an ordered set of vertices $p_{1}, \ldots, p_{n}$ in the plane, a set of orientations $\mathcal{C}$, and a constant $\epsilon$, we search for a " $\mathcal{C}$-oriented" polygonal chain $Q$ consisting of the minimum number of line segments that has distance at most $\epsilon$ to $P$ in the Fréchet metric. A polygonal chain is $\mathcal{C}$-oriented if the line segments are parallel to orientations in $\mathcal{C}$. We restrict our attention to the version of the problem where two circles of radius $\epsilon$ formed around adjacent vertices of the polygonal chain do not intersect. We solve the $\mathcal{C}$-oriented line simplification problem constructively by using dynamic programming together with a nice data structure. For usual cases of $\mathcal{C}$ our algorithm solves the problem in time $\mathcal{O}\left(k n^{2} \log (n)\right)$ where $k$ is the minimum number of line segments of $Q$ and uses $\mathcal{O}\left(k n^{2}\right)$ space.
\end{abstract}

\section{Introduction}

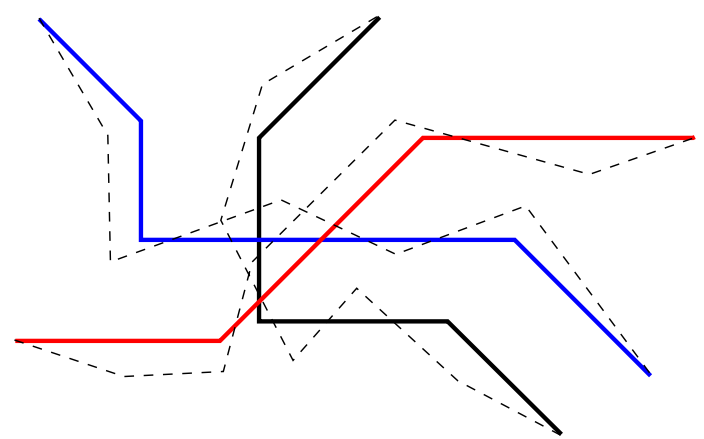

Figure 1: Subway lines (dashed) and $\mathcal{C}^{-}$ oriented simplified subway lines $(|\mathcal{C}|=4)$.

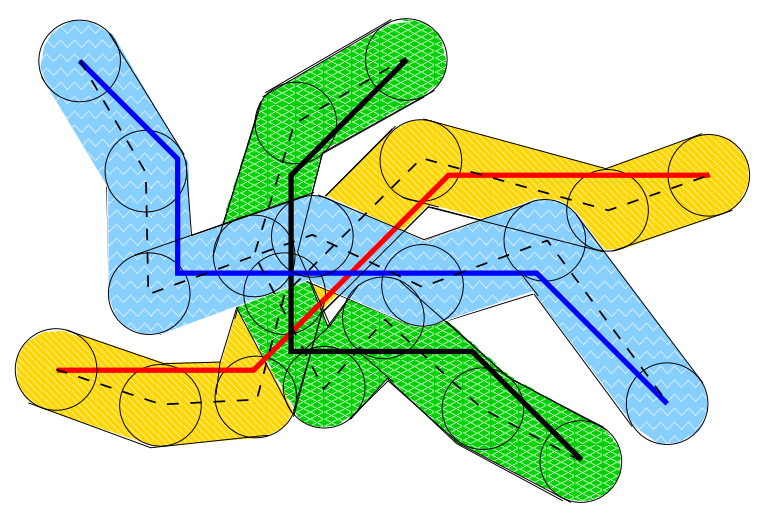

Figure 2: The $\epsilon$-area around each subway line is shaded.

Maps such as those used to describe subway lines, bus plans, or cartographic schemas for gas, water or electricity mains are often drawn $\mathcal{C}$-oriented. Each line segment in these plans is parallel to an orientation in a fixed set of orientations (vectors) $\mathcal{C}$ in $\mathbb{R}^{2}$. We assume that for each $c \in \mathcal{C}$ the orthogonal orientation to $c$, denoted as $\bar{c}$, is element of $\mathcal{C}$. This restriction is trivially

\footnotetext{
${ }^{\dagger}$ This work was partially supported by grants from the Swiss Federal Office for Education and Science (Projects ESPRIT IV LTR No. 21957 CGAL and N0. 28155 GALIA), and by the Swiss National Science Foundation (grant "Combinatorics and Geometry").

${ }^{\ddagger}$ Institute for Theoretical Computer Science, ETH Zurich, Switzerland, email:neyer@@inf.ethz.ch
} 
fulfilled for an even number of uniformly distributed orientations. Requiring the maps to be $\mathcal{C}$-oriented helps to make the maps look graphically clearer, more structured, and hence easier to read. Consider, for example, the creation of a subway line map given a city map. Typically, the number of $\mathcal{C}$-orientations used would be 4 : horizontal, vertical, and both diagonals. The subway line normally maintains geographical informations like "is in the north of", "crosses", etc. We restrict attention to one central task in the automatic generation of $\mathcal{C}$-oriented maps: the $\mathcal{C}$-oriented line simplification problem.

Let $p_{1}, p_{2}, \ldots, p_{n}$ be $n$ vertices in the plane. Let $\overline{p_{j} p_{j+1}}$ be a (closed) line segment from $p_{j}$ to $p_{j+1}$. We also call $\overline{p_{j} p_{j+1}}$ a link. We denote a polygonal chain $P$ by $P=\left\langle p_{1}, \ldots, p_{n}\right\rangle$, it consists of line segments $\overline{p_{j} p_{j+1}}, 1 \leq j<n$. In the $\mathcal{C}$-oriented line simplification problem we want to compute a $\mathcal{C}$-oriented polygonal chain (i.e. each of the links is $\mathcal{C}$-oriented) with a minimum number of links that represents $P$ "well". Representing $P$ well means that the approximation leads along $P$ and remains within $\epsilon$-distance of $P$, where $\epsilon$ is a constant quality bound.

Generalization and especially line simplification are well studied problems in cartography. See [Wei97] for a survey on cartographic generalization requirements and techniques and [AG95, AG96] for a survey on geometric techniques that have been used to measure similarity or distance between shapes. Probably the most popular (but not $\mathcal{C}$-oriented) line simplification algorithm is the Douglas Peucker Algorithm [DP73] and its improvement [HS92]. When the simplification line must also be $\mathcal{C}$-oriented, the Douglas Peucker algorithm can not be applied directly because the line formed from two consecutive points in the line simplification may not be parallel to an orientation in $\mathcal{C}$; the Douglas Peucker algorithm uses only such original data points. Guibas et.al. solved the (non $\mathcal{C}$-oriented) line simplification problem under several optimization criteria [GHMS93]. They give an $\mathcal{O}\left(n^{2} \log n\right)$ time algorithm for finding an approximation that consists of a minimum number of links that has Fréchet distance at most $\epsilon$ to the original polygonal chain. In case that the $\epsilon$-circles around adjacent vertices of the original polygonal chain do not intersect, they provide a linear time algorithm. Adegeest et.al. give a $\mathcal{O}\left(c^{2} n \log ^{2} n\right)$ time algorithm for computing minimum c-oriented link paths between a pair of points in the plane that avoids a set of $n$ obstacles [AOS94]. In their definition of c-orientation $\mathrm{c}$ is a number greater or equal to two defining $c$ unit vectors where each pair of clockwise adjacent vectors has the angle $\frac{\pi}{c}$. For a given starting point $A$, they iteratively determine the sets of all points that can be reached by paths of one link, two links, etc. This is the first approach solving the $\mathcal{C}$-oriented line simplification. We use ideas from [GHMS93] and [AOS94], but we note that both methods can not be applied without major adjustments.

\section{Preliminaries and Problem Definition}

In this section we first introduce some necessary notations and definitions. Then, we formalize the $\mathcal{C}$-oriented line simplification problem.

For two points $p$ and $q$ we denote $\vec{p} \vec{q}$ as the vector $(q-p)$. We distinguish between points and vertices of a polygonal chain: we call any $q \in \overline{p_{j} p_{j+1}}$ a point of $P$ and each $p_{j}$ a vertex, $1 \leq j \leq n$. Let $p$ and $q$ be two points on a simple polygon $P$. If $p$ precedes $q$ on $P$ we write $p \prec q$. 


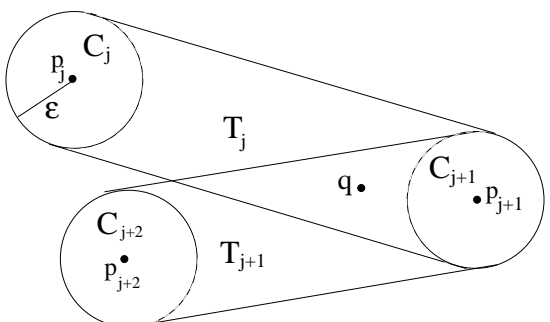

Figure 3: Tube $T_{j}, T_{j+1}$.

Let $P=\left\langle p_{1}, \ldots, p_{n}\right\rangle$ be a polygonal chain. Call a circle with radius $\epsilon$ and center $p_{j}$ the $\epsilon$-circle $C_{j}$ of $p_{j}$. The convex hull of two $\epsilon$-circles $C_{j}$ and $C_{j+1}$ from two consecutive vertices $p_{j}$ and $p_{j+1}$ is called the tube $T_{j}$. Figure 3 shows two tubes $T_{j}, T_{j+1}$, and a point $q$ lying in both tubes but not in the $\epsilon$-circle they share. We need to distinguish between $q \in T_{j}$ and $q \in T_{j+1}$. Therefore, to a tube $T_{j}$ including the link $\overline{p_{j} p_{j+1}}$ we assign a level $j$, and to a $\epsilon$ circle $C_{j}$ including its center $p_{j}$ we assign levels $j$ and $j-1$, if $j>1$. All operations like $\cap, \epsilon, \prec$ now refer to their operands and their level. As a consequence $P$ is simple, $T_{j} \cap T_{j+1}=C_{j+1}$, and $T_{j} \cap T_{j+l}=\emptyset$, for $l>1$. Let $\mathcal{T}=\bigcup_{j=1}^{n-1} T_{j}$. (Figure 2 shows a set of polygonal chains with the area of $\mathcal{T}$ shaded gray.) Clearly, each point in $\mathcal{T}$ has distance at most $\epsilon$ to the polygonal chain. We require that the approximation lies in $\mathcal{T}$, starts at a point in a start set $S \subseteq C_{1}$, ends at a point in an end set $E \subseteq C_{n}$, uses only orientations in $\mathcal{C}$, and leads through consecutive tubes.

\section{Problem 2.1 ( $\mathcal{C}$-oriented line approximation)}

Instance: Let $P=\left\langle p_{1}, \ldots, p_{n}\right\rangle$ be a polygonal chain in $\mathbb{R}^{2}$, let $\mathcal{C}$ be a set of orientations, $\epsilon$ a positive constant, such that $C_{j} \cap C_{j+1}=\emptyset, j=1, \ldots, n-1, S \subseteq C_{1}$ a connected set of possible start points and $E \subseteq C_{n}$ a set of possible end points.

Problem: Find a $\mathcal{C}$-oriented line approximation of $P$. That is a $\mathcal{C}$-oriented polygonal chain $Q=\left\langle q_{1}, \ldots, q_{m}\right\rangle$, such that $q_{1} \in S, q_{m} \in E$, and $q_{i} \in \mathcal{T}, i=1, \ldots, m$. Furthermore, link $\overline{q_{i} q_{i+1}}$ intersects all $C_{j+1}, \ldots, C_{l}$ in this order, in case $q_{i} \in T_{j}, q_{i+1} \in T_{l}, j$ maximal, $l$ minimal, and $j<l$. In case that $q_{i} \in T_{l}, q_{i+1} \in T_{j}, j$ maximal, $l$ minimal, and $j<l$, link $\overline{q_{i} q_{i+1}}$ intersects all $C_{l}, \ldots, C_{j+1}$ in this order.

\section{Problem 2.2 (C-oriented line simplification $(\mathcal{C} O L S)$ )}

Instance: An instance of the $\mathcal{C}$-oriented line approximation problem.

Problem: Find a $\mathcal{C}$-oriented line approximation of $P$ that consists of a minimum number of links.

\section{Reachable Regions}

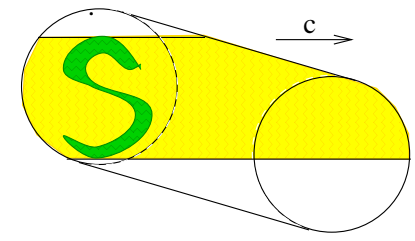

Figure 4: Horizontal reachable region of $S$ in a tube (shaded gray).
In this section we introduce the concept of reachable regions, modified from [AOS94]. For any tube $T_{j}$ and any number of links $i$ we will define a set that contains all points $q \in T_{j}$ for which a $\mathcal{C}$-oriented link path exists that starts in $S$ and leads through $T_{1}, \ldots, T_{j}$. For these sets we define a recursion formula that can be calculated in a dynamic program.

Let $p$ and $q$ be two points. We say $p$ reaches $q$ w.r.t. $c$ if there exists a $\lambda \in \mathbb{R}$ such that $q=p+\lambda c$. Locally in a tube we define the set of points that are reachable with one single link within a single tube as follows. 
Definition 3.1 (Reachable region $\mathcal{R} \mathcal{R}(S, c)$ ) Let $T$ be a tube, $S \subseteq T$, and $c \in \mathcal{C}$. We denote by $\mathcal{R} \mathcal{R}(S, c)$ the set of all points $q \in T$ for which there exists a point $p \in S$ such that $p$ reaches $q$ with respect to c. For $\bigcup_{c \in \mathcal{C}} \mathcal{R} \mathcal{R}(S, c)$ we simply write $\mathcal{R} \mathcal{R}(S)$.

Figure 4 gives an example of a reachable region. Now, we extend the notion of a reachable region to allow multiple links within a sequence of tubes. Note that the following definition is consistent with the definition of Problem 2.1.

Definition 3.2 (Reachable region $\mathcal{R} \mathcal{R}^{i}\left(S, T_{j}\right)$ ) Let $T_{j}$ be the $j^{\text {th }}$ tube of a polygonal chain $P$ and $S$ the start set. We denote by $\mathcal{R} \mathcal{R}^{i}\left(S, T_{j}\right)$ the set of all points $q \in T_{j}$ for which a $\mathcal{C}$-oriented i-link path $Q=\left\langle q_{1}, \ldots, q_{i}\right\rangle$ from $S$ to $q$ exists such that $q_{l} \in \mathcal{T}, l=1, \ldots, i$. Furthermore, link $\overline{q_{l} q_{l+1}}$ intersects all $C_{j+1}, \ldots, C_{d}$ in this order, in case that $q_{l} \in T_{j}, q_{l+1} \in T_{d}, j$ maximal, $d$ minimal, and $j<d$. In case that $q_{l} \in T_{d}, q_{l+1} \in T_{j}, j$ maximal, $d$ minimal, and $j<d$, link $\overline{q_{l} q_{l+1}}$ intersects all $C_{d}, \ldots, C_{j+1}$ in this order.

Note that $\mathcal{R R}^{0}\left(S, T_{1}\right)=S, \mathcal{R}^{0}\left(S, T_{j}\right)=\emptyset$ for $j>1$ and $\mathcal{R} \mathcal{R}^{1}\left(S, T_{1}\right)=\mathcal{R} \mathcal{R}(S)$ in respect to tube $T_{1}$. Throughout this paper we measure the distance between two points $p=\left(x_{1}, y_{1}\right)$ and $q=\left(x_{2}, y_{2}\right)$ in $\mathbb{R}^{2}$ in the $L_{2}$ metric. That is, $\|p-q\|_{2}:=\left(\left(x_{1}-x_{2}\right)^{2}+\left(y_{1}-y_{2}\right)^{2}\right)^{\frac{1}{2}}$. In the next lemma and theorem we prove that $\mathcal{C}$ OLS always admits a solution.

Lemma 3.3 (Existence) Let $p, q \in T_{1}$, there exists a nonnegative integer $k$ such that $q \in$ $\mathcal{R R}^{k}\left(p, T_{1}\right) . k$ is bounded by $\frac{\|p-q\|_{2}}{2 \epsilon}+4$.

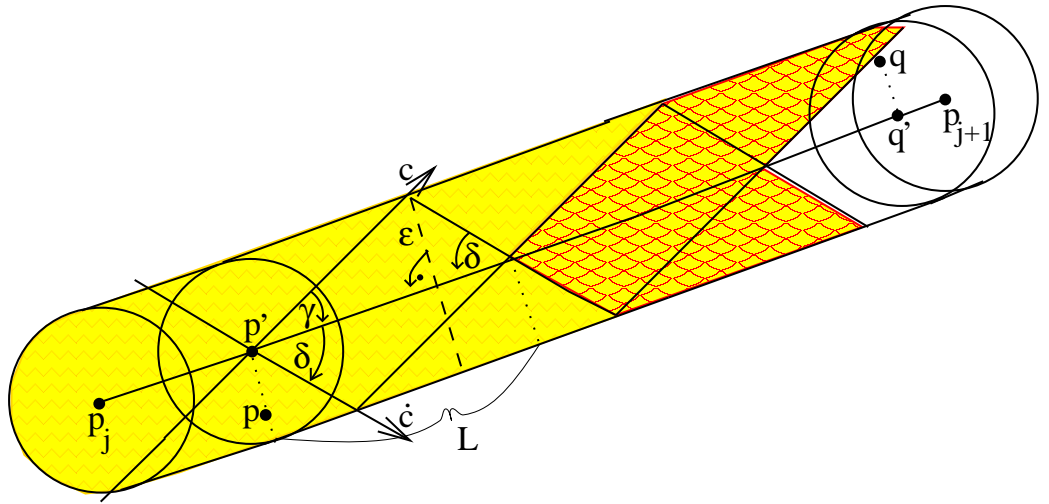

Figure 5: There exists a $\mathcal{C}$-oriented link path consisting of a bounded number of links between each pair of points in a tube.
Proof: If $p=q$ we may choose $k=0$. Assume henceforth that $p \neq q$. There exists a point $p^{\prime}$ along $\overline{p_{j} p_{j+1}}$ such that $\left\|p-p^{\prime}\right\|_{2} \leq \epsilon$. Analogously, there exists a point $q^{\prime}$ along $\overline{p_{j} p_{j+1}}$ such that $\left\|q-q^{\prime}\right\|_{2} \leq \epsilon$. $p$ lies in an $\epsilon$-circle $C^{\prime}$ around $p^{\prime}$ which is contained in $T_{1}$. If $\overrightarrow{p p^{\prime}} \in \mathcal{C}$ then $p$ can be reached with one link. Otherwise, $p$ lies in the wedge of 2 orientations $c$ and $\dot{c}$ in $\mathcal{C}$ with respect to $\overrightarrow{p p^{\prime}}$. Since the angle $\varangle(c, \dot{c}) \leq \frac{\pi}{2}$, there exists a $\lambda, \nu \in \mathbb{R}$ such that $p=p^{\prime}+\lambda c+\nu \dot{c}$, where the links $\overline{p^{\prime}\left(p^{\prime}+\lambda c\right)}$ and $\overline{\left(p^{\prime}+\lambda c\right) p}$ lie inside $C^{\prime}$.

Let $\overline{p^{\prime} q^{\prime}}$ be in the wedge of $c$ and $\dot{c}, c, \dot{c} \in \mathcal{C}$ (see Figure 5). We now show that there exists a $\mathcal{C}$-oriented link path from $p^{\prime}$ to $q^{\prime}$ inside $T_{1}$ using only the two orientations $c$ and $\dot{c}$. Let $\gamma$ be the angle between $c$ and $\overrightarrow{p^{\prime} q^{\prime}}$. Let $\delta$ be the angle between $\dot{c}$ and $\overrightarrow{p^{\prime} q^{\prime}}$. Starting at $p^{\prime}$ using only directions $c$ and $\dot{c}$ one can reach all points on the lines $p^{\prime}+\lambda c$ and $p^{\prime}+\lambda \dot{c}$ that lie inside $T_{1}$, $\lambda \in \mathbb{R}$. With one more link, all points in the light shaded region are reachable. With two more links also the points in the dark shaded region are reachable and so on. Within each additional link an additional length of length $L=2 \epsilon(\cot \gamma+\cot \delta) \operatorname{can}$ be reached on $\overline{p^{\prime} q^{\prime}}$. Since $\gamma+\delta \leq \frac{\pi}{2}$ it follows that $L \geq 2 \epsilon$. Thus the number of links that are necessary to reach $q^{\prime}$ from $p^{\prime}$ is bounded by $\frac{\left\|p^{\prime}-q^{\prime}\right\|_{2}}{2 \epsilon}$. Therefore, $q \in \mathcal{R}^{k}\left(p, T_{1}\right)$, with $k=\frac{\|p-q\|_{2}}{2 \epsilon}+4$. 
Observation 3.4 Every polygonal chain $P$ has a $\mathcal{C}$-oriented line simplification for arbitrary $\epsilon>0$.

Proof: Let $S$ be the set of start points in $C_{1}$. Lemma 3.3 yields that for any point $q \in C_{2}$ there exists a $i \in I N$ such that $q \in \mathcal{R} \mathcal{R}^{i}\left(S, T_{1}\right)$. Repetitive application of this observation yields that for any point $q \in T_{j}$ there exists a $i$ such that $q \in \mathcal{R} \mathcal{R}^{i}\left(S, T_{j}\right), j=1, \ldots, n$. In particular, for each $q \in E$ there exists a $i \in I N$ such that $q \in \mathcal{R} \mathcal{R}^{i}\left(S, T_{n-1}\right)$. Let $Q$ be the according $\mathcal{C}$-oriented link path. From the definition of the reachable regions $\mathcal{R} \mathcal{R}^{i}\left(S, T_{j}\right)$ it follows that the path is a $\mathcal{C}$-oriented approximation of $P$. From the existence of a $\mathcal{C}$-oriented line approximation of $P$ follows the existence of a $\mathcal{C}$-oriented line approximation consisting of a minimum number of links.

We now introduce the concept of extensions. This allows us to extend a reachable region $r \subseteq \mathcal{R} \mathcal{R}^{i}\left(S, T_{k}\right)$ into $\mathcal{R} \mathcal{R}^{i}\left(S, T_{j}\right)$ whenever possible, $k \neq j$. That is, we compute the $c$-oriented reachable region $r$ of a $\dot{c}$-oriented reachable region $r^{\prime}, c \neq \dot{c}$, and try to extend it into as many consecutive tubes as possible. Figure 6 shows an extension of a $\dot{c}$-oriented reachable region from $T_{j-1}$ to $T_{j}$. Note that the extension in Figure 7 is bounded by a boundary segment of $C_{j}$. A point $q$ in the striped black region of Figure 7 does not belong to the extension of $r^{\prime}$ from $T_{j-1}$ to $T_{j}$, since a link from $r^{\prime}$ into that region does not cut $C_{j}$.

We define a forward extension as an extension from a tube $T_{k}$ into a tube $T_{j}$ with $k<j$. Similarly, a backward extension is an extension from a tube $T_{j}$ into a tube $T_{k}$ with $j>k$.

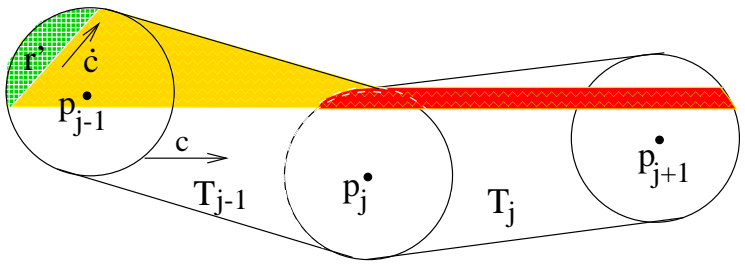

Figure 6: The $\dot{c}$-oriented reachable region $r^{\prime}$ is extended (shaded dark).

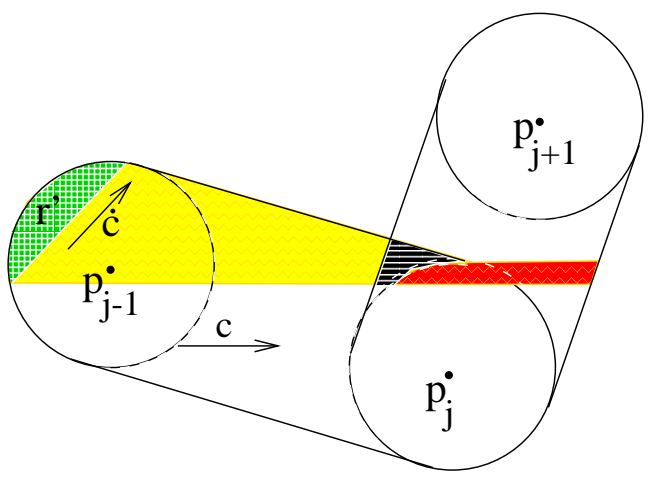

Figure 7: The extension of $r^{\prime}$ is bounded by a segment of $C_{j}$ in (shaded dark).

\section{Definition 3.5 (Extension)}

Forward Extension $\mathcal{F E}\left(U, T_{k}, T_{j}\right):$ Let $U \subseteq T_{k}$ for some $k<j$. We denote by $\mathcal{F E}\left(U, T_{k}, T_{j}\right)$ the set of all points $q \in T_{j}$ for which a $\mathcal{C}$-oriented link from a point $p \in U \backslash C_{k+1}$ exists, such that $\overline{p q}$ intersects all $C_{k+1}, \ldots, C_{j}$ in this order. With $\mathcal{F E}\left(U, T_{k}, T_{j}, c\right)$ we denote the subset of $\mathcal{F E}\left(U, T_{k}, T_{j}\right)$ where all links are c-oriented, $c \in \mathcal{C}$. Then, $\mathcal{F E}\left(U, T_{k}, T_{j}\right)=$ $\bigcup_{c \in \mathcal{C}} \mathcal{F} \mathcal{E}\left(U, T_{k}, T_{j}, c\right)$ follows.

Backward Extension $\mathcal{B E}\left(U, T_{k}, T_{j}\right)$ : Let $U \subseteq T_{j}$ for some $k<j$. We denote by $\mathcal{B E}\left(U, T_{k}, T_{j}\right)$ the set of all points $q \in T_{k} \backslash C_{k+1}$ for which a $\mathcal{C}$-oriented link from a point $p \in U$ exists, such that $\overline{p q}$ intersects all $C_{j}, \ldots, C_{k+1}$ in this order. 
We will show in the next lemma that we do not need backward extensions to reach all reachable points with a minimum number of links. This does not follow directly from the problem definition. We have to make use of the assumption that for each orientation $c$ in $\mathcal{C}$ also the orthogonal orientation $\bar{c}$ is element of $\mathcal{C}$.

Lemma 3.6 (Backward Extensions) Let $q \in \mathcal{R R}^{i}\left(S, T_{j}\right)$. Then, there exists a $\mathcal{C}$-oriented path $Q=\left\langle q_{1}, \ldots, q_{i}=q\right\rangle$ from $q_{1} \in S$ to $q$ such that each link $\overline{q_{k} q_{k+1}}$ is either contained in a tube $T_{l}$ for some $l \leq j$, or $\overline{q_{k} q_{k+1}}$ intersects all $C_{e+1}, \ldots, C_{f}$ in this order with $q_{k} \in T_{e}$, $q_{k+1} \in T_{f}$, e maximal, $f$ minimal, and $e<f$.

Proof: Assume there exists a $q \in \mathcal{R R}^{i}\left(S, T_{j}\right)$ that can only be reached with $i$ links, when backward extensions are also considered. Let $Q=\left\langle q_{1}, \ldots, q_{i}=q\right\rangle$ be a path from $q_{1} \in S$ to $q$. Then, there exists a minimum $k$, a maximum $e$, and a minimum $f$ with $e<f, q_{k} \in T_{e}$, $q_{k+1} \in T_{f}$ such that $\overline{q_{k} q_{k+1}}$ intersects $C_{f}, \ldots, C_{e+1}$ in this order. We now show how this link,

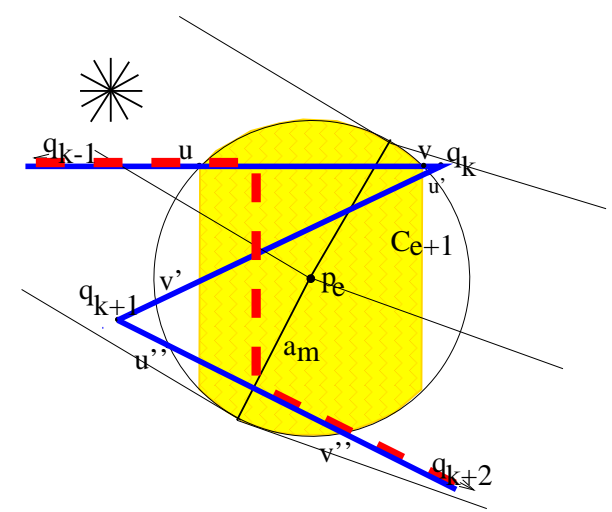

Figure 8: The solid polygonal subchain can be replaced by the dashed one.

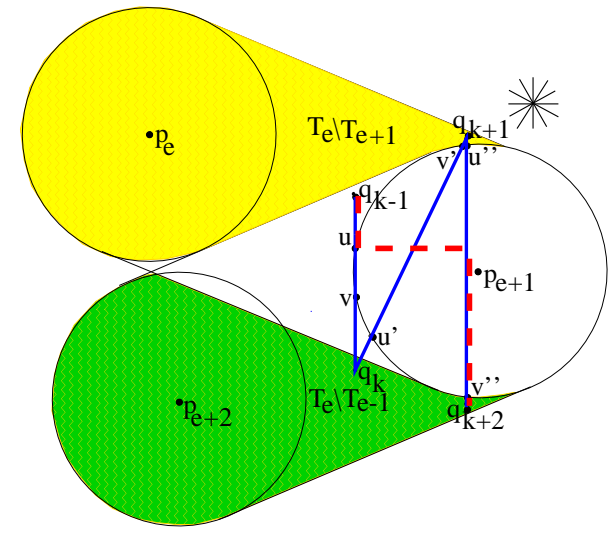

Figure 9: The solid polygonal subchain can be replaced by the dashed one.

its predecessor link, and successor link can be replaced by 3 other $\mathcal{C}$-oriented links that are not backward extensions. Observe that $Q$ has no self intersections, when we assign to a tube $T_{i}$ a level $i$ and to a circle $C_{i}$ level $i-1$ and level $i$, as introduced in Section 2.

Note that $Q$ enters the circle $C_{e+1}$ three times. Let the intersecting points of $Q \cap \partial C_{e+1}$ be $u \prec v \prec u^{\prime} \prec v^{\prime} \prec u^{\prime \prime} \prec v^{\prime \prime}$ (see Figure 8, 9). Since $u$ can reach $v^{\prime \prime}$ within $C_{e+1}$ in at most 2 links we can assume that $q_{k-1}$ lies in a tube $T_{x}$ with $x \leq e$ and $q_{k+2}$ lies in a tube $T_{y}$ with $y>e$. Therefore, $\overline{u v}, \overline{u^{\prime} v^{\prime}}$, and $\overline{u^{\prime \prime} v^{\prime \prime}}$ are segments of $Q$ and therefore $\mathcal{C}$-oriented. Let $\overline{u v}$ have orientation $c$ and let $\overline{u^{\prime \prime} v^{\prime \prime}}$ have orientation $\dot{c}$.

We make a case distinction according to the location of $u, v, u^{\prime \prime}$ and $v^{\prime \prime}$ :

There exists a line $a_{m}$ that cuts $p_{e+1}, \overline{u v}$ and $\overline{u^{\prime \prime} v^{\prime \prime}}$ : In this case we claim that there exists a $p \in \overline{u v}$ such that $p+\lambda \bar{c}$ has non empty intersection with $\overline{u^{\prime \prime} v^{\prime \prime}}$. Let $R=\left\{x \in C_{e+1} \mid \exists y \in\right.$ $\overline{u v}, \lambda \in \mathbb{R}$ such that $x=y+\lambda \bar{c}$ \}. Observe that $R$ is point symmetric to the midpoint $p_{e+1}$. Since $u$ and $v$ are positioned on opposite sides of $a_{m}$ in $C_{e+1}$, it follows that each point on $a_{m}$ can be reached with an $\bar{c}$-oriented link starting at a point on $\overline{u v}$. Since $\overline{u^{\prime \prime} v^{\prime \prime}}$ cuts $a_{m}$ it follows that there exists a point $z$ on $\overline{u v}$, a point $z^{\prime}$ on $\overline{u^{\prime \prime} v^{\prime \prime}}$, and a $\lambda \in \mathbb{R}$ with $z^{\prime}=z+\lambda \bar{c}$. Thus, replacing $Q$ by $Q^{\prime}=\left\langle q_{1}, \ldots, q_{k-1}, z, z^{\prime}, q_{k+2}, \ldots, q_{i}\right\rangle$ yields that $Q^{\prime}$ has as many links as $Q$ and $\left\langle q_{1}, \ldots, q_{k-1}, z, z^{\prime}, q_{k+2}\right\rangle$ has no backward extension. 
The points $u, v, u^{\prime \prime}$ and $v^{\prime \prime}$ lie on one half circle $H$ of $\partial C_{e+1}$ : We make a case distinction according to the possible orders of $u, v, u^{\prime \prime}$, and $v^{\prime \prime}$ on $H$ :

Order $u^{\prime \prime}, u, v, v^{\prime \prime}$ and $u, u^{\prime \prime}, v^{\prime \prime}, v$ and their reversals: See Figure 9 for an example. Then, either there exists a $\lambda \in \mathbb{R}$ and a $z$ on $\overline{u^{\prime \prime} v^{\prime \prime}}$ such that $z=u+\lambda \bar{c}$ or there exists a $\lambda \in \mathbb{R}$ and a $z$ on $\overline{u v}$ such that $z=u^{\prime \prime}+\lambda \overline{\dot{c}}$. In the first case, $Q^{\prime}=\left\langle q_{1}, \ldots, q_{k-1}, u^{\prime}, z, q_{k+2}, \ldots, q_{i}\right\rangle$ is a link path with at most as many links as $Q$ has and $\left\langle q_{1}, \ldots, q_{k-1}, u^{\prime}, z, q_{k+2}\right\rangle$ has no backward extension. In the second case $Q^{\prime}=\left\langle q_{1}, \ldots, q_{k-1}, z, u^{\prime \prime}, q_{k+2}, \ldots, q_{i}\right\rangle$ is a link path with at most as many links as $Q$ has and $Q^{\prime}=\left\langle q_{1}, \ldots, q_{k-1}, z, u^{\prime \prime}, q_{k+2}\right\rangle$ has no backward extension.

Order $u, v, v^{\prime \prime}, u^{\prime \prime}$, order $v, u, u^{\prime \prime}, v^{\prime \prime}$, and their reversals: In this case either link $\overline{q_{u} q_{k}}$ lies inside $T_{e}$ or $\overline{q_{k+1} q_{k+2}}$ lies inside $T_{e}$. In both cases $q_{k+1}$ can be reached without backward extensions.

This procedure can be applied to all backward extensions in $Q$. Thus, all points in $\mathcal{R} \mathcal{R}^{i}\left(S, T_{j}\right)$ are reachable without backward extensions.

We now can express the calculation rule for the reachable regions as a recursion formula.

Theorem 3.7 (Recursion Formula) For $i, j>0$ we have

$$
\mathcal{R R}^{i}\left(S, T_{j}\right)=\mathcal{R} \mathcal{R}\left(\mathcal{R} \mathcal{R}^{i-1}\left(S, T_{j}\right)\right) \cup \bigcup_{k=1}^{j-1} \mathcal{F} \mathcal{E}\left(\mathcal{R} \mathcal{R}^{i-1}\left(S, T_{k}\right), T_{k}, T_{j}\right)
$$

With $\mathcal{R} \mathcal{R}^{i}\left(S, T_{j}, c\right)$ we denote the subset of $\mathcal{R} \mathcal{R}^{i}\left(S, T_{j}\right)$ where the last link is c-oriented. Then, $\mathcal{R}^{i}\left(S, T_{j}\right)=\bigcup_{c \in \mathcal{C}} \mathcal{R} \mathcal{R}^{i}\left(S, T_{j}, c\right)$ follows.

Proof: In the previous lemma we showed that backward extensions are not necessary to reach a point within a minimum number of $\mathcal{C}$-oriented links. Thus, the correctness of this formula follows.

Corollary 3.8 Let $i$ be minimal such that $E \cap \mathcal{R} \mathcal{R}^{i}\left(S, T_{n-1}\right) \neq \emptyset$. The minimum $\mathcal{C}$-oriented link path from $S$ to $E$ has i links.

Proof: With Theorem 3.7 it follows that the corresponding $\mathcal{C}$-oriented $i$-link path for each $q \in\left(E \cap \mathcal{R}^{i}\left(S, T_{n-1}\right)\right)$ consists of a minimum number of links.

The sets $\mathcal{R R}^{i}\left(S, T_{j}\right)$ can be computed by dynamic programming according to the Recursion Formula (see Algorithm 1). Let $q \in \mathcal{R}^{i}\left(S, T_{j}, c\right)$. The $\mathcal{C}$-oriented link path from $q$ to $S$ can be computed as follows: Starting at $q$ we create a line $q+\lambda c, \lambda \in \mathbb{R}$. The $c$-oriented reachable region of $q$ is associated with its generating reachable region $r^{\prime} \in \mathcal{R R}^{i-1}\left(S, T_{k}, \dot{c}\right)$. We compute a point $q^{\prime} \in r^{\prime}$ which lies on $q+\lambda c$. This point $q^{\prime}$ is the next vertex of the link path. Then, we repeat this procedure starting at $q^{\prime}$ and continue until we reach a point in $S$.

Inserting each reachable region into a set $\mathcal{R R}^{i}\left(S, T_{j}\right)$ yields that each region $r \in \mathcal{R} \mathcal{R}^{i}\left(S, T_{j}\right)$ generates at most $|\mathcal{C}|$ reachable regions in $\mathcal{R} \mathcal{R}^{i+1}\left(S, T_{j}\right)$. Furthermore, each region $r \in \mathcal{R} \mathcal{R}^{i}\left(S, T_{j}\right)$ can generate at most $|\mathcal{C}|$ reachable regions in the extension step per tube $l=j+1, \ldots n$. Let $f(i, j)$ be the number of reachable regions in $\mathcal{R}^{i}\left(S, T_{j}\right), 1 \leq j \leq n-1$. The following equations hold:

$$
f(i, j) \leq \begin{cases}|\mathcal{C}| \sum_{l=1}^{j} f(i-1, l) & \text { if } i>1, j>1 \\ |\mathcal{C}| & \text { if } i=1, j \geq 1\end{cases}
$$

These recurrence equations lead to a number of reachable regions that grows exponentially in the number of line segments $k$ of the minimum link path. 


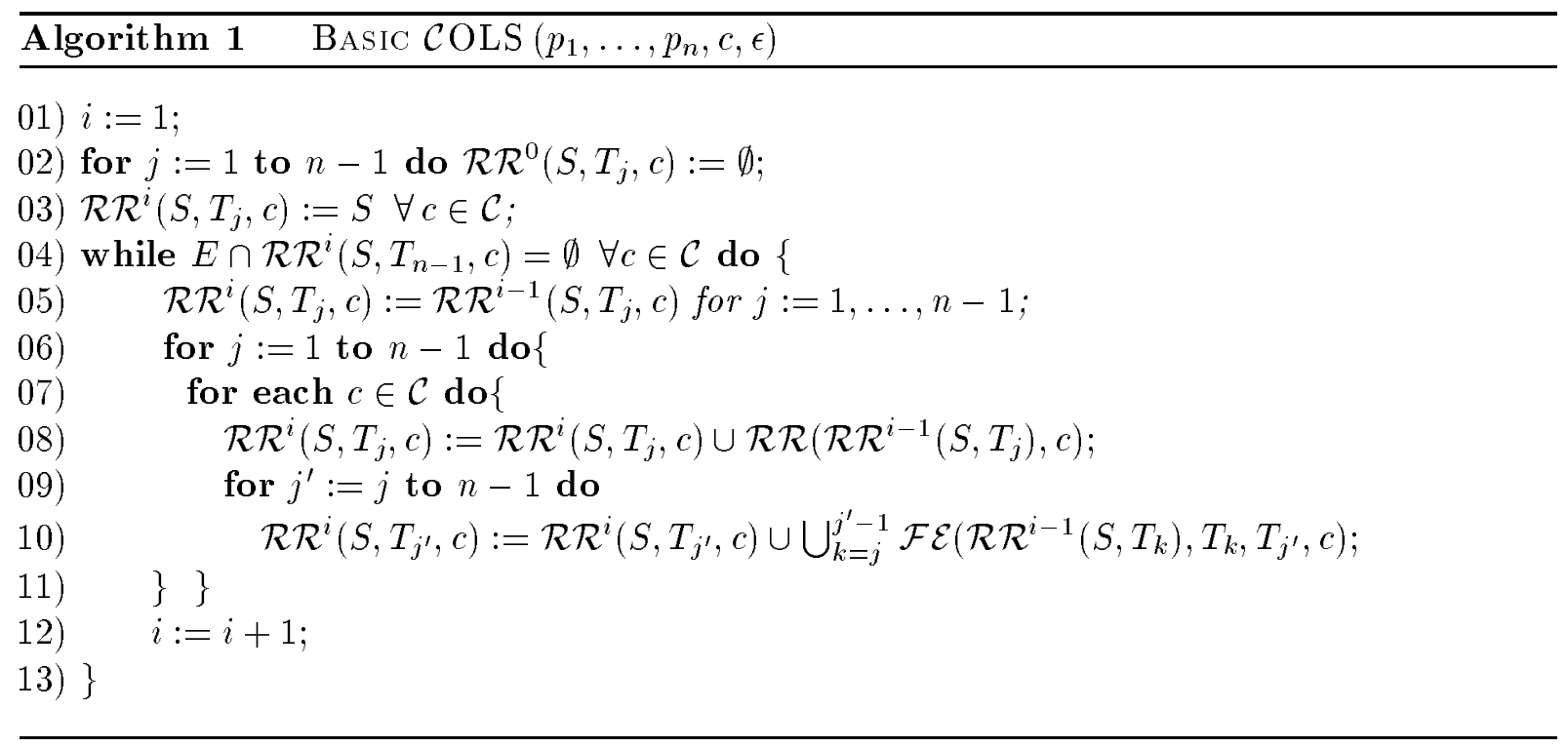

\section{Speed-Up Techniques}

In order to consider only a polynomial (in $n, k$, and $|\mathcal{C}|$ ) number of reachable regions we study the form of reachable regions and classify them. This allows us to handle and store the reachable regions efficiently.

Lemma 4.1 Let $r$ be a reachable region in $\mathcal{R}^{i}\left(S, T_{j}\right)$. Then, $r \cap C_{j} \neq \emptyset$.

Proof: This follows inductively. The reachable region of $\mathcal{R R}^{1}\left(S, T_{1}, c\right)$ has nonempty intersection with $C_{1}$ since $S \subseteq C_{1}, c \in \mathcal{C}$. Therefore $\mathcal{R} \mathcal{R}^{i}\left(S, T_{1}, c\right) \cap C_{1} \neq \emptyset$, for any $c \in \mathcal{C}$. Let $r \in \mathcal{R R}^{i}\left(S, T_{k}\right)$ be a reachable region in $T_{k}, k<j$. Since the extension $\mathcal{F E}\left(r, T_{k}, T_{j}, c\right)$ has nonempty intersection with $C_{j}$ (by definition) the same follows for any reachable region in $\mathcal{R}^{i}\left(S, T_{j}\right), 1 \leq j \leq n-1$.

Let $r$ be a $c$-oriented reachable region in a tube $T_{j}$. Then, the boundary of $r$ has the nice property that is determined by the boundary of $T_{j}$, the boundary of $C_{j}$ and $c$-oriented lines. In case that $C_{j} \cap C_{j+1} \neq \emptyset$ the boundary of a reachable region can also contain boundary segments of non local circles, which would make the handling much more difficult.

Theorem 4.2 (Type of a Reachable Region) Let $S \subseteq C_{1}$ be a connected set of points in $C_{1}$. Let $c \in \mathcal{C}$. A c-oriented reachable region $r$ in $T_{j}$ can be categorized to have the following forms:

1. $r$ is determined by the intersection of two parallel c-oriented lines and the boundary of $T_{j}$. We say that type $(r)=1$. (See Figure 6 .)

2. $r$ is a reachable region in $T_{j}$ that is defined by a connected boundary segment $s_{j} \subseteq$ $\partial C_{j} \backslash \partial T_{j-1}$ and a direction $\tau \in\{c,-c\} . r=\left\{q \in T_{j} \mid \exists q^{\prime} \in s_{j}, \lambda>0\right.$ such that $\left.q=q^{\prime}+\lambda \tau\right\}$. We say that type $(r)=2$. (See Figure 7.)

Proof: Let $r$ be the $c$-oriented reachable region generated from $r^{\prime}$ in $T_{j}$. We show by induction on $i$ that each reachable region $r \in \mathcal{R}^{i}\left(S, T_{j}, c\right)$ is of type 1 or type 2 .

$\underline{i=1, j=1:}$ The $c$-oriented reachable region of $S$ in $T_{1}$ clearly is of type 1 (see also Figure 4 ). 
$i=1, j>1: r=\mathcal{F E}\left(r^{\prime}, T_{1}, T_{j}, c\right)$. Let $s=\partial C_{j} \backslash \partial T_{j-1} \cap r$. Clearly, for each $q \in r$ there exists a $q^{\prime} \in s$ and a $\lambda \in \mathbb{R}$ such that $q=q^{\prime}+\lambda c$ and the line $\overline{q^{\prime} q} \cap s \neq \emptyset$. For each pair $\left(q, q^{\prime}\right)$ with $q \in r$ and $q^{\prime} \in s$ such that $q=q^{\prime}+\lambda c$, the sign of $\lambda$ is either positive or negative since $C_{j} \cap C_{j+1}=\emptyset$. Let $\tau=c$ if $\lambda>0, \tau=-c$ otherwise. Therefore, $r=\mathcal{F E}\left(r^{\prime}, T_{1}, T_{j}, c\right)=\left\{q \in T_{j} \mid \exists q^{\prime} \in s, \lambda>0\right.$ such that $\left.q=q^{\prime}+\lambda \tau\right\} . s$ is a connected boundary segment since for each two points $q_{1}$ and $q_{2}$ in $s$ with $q_{1}+\lambda \tau$ intersects $s$ and $q_{2}+\lambda \tau$ intersects $s$ there is a path from $q_{1}$ to $q_{2}$ in $s$ and all points $q_{3}$ on this path fulfill that $q_{3}+\lambda \tau$ also intersects $\partial C_{j} \backslash \partial T_{j-1}$ and therefore $s$. Since $s=\partial C_{j} \backslash \partial T_{j-1} \cap r, s$ can be at most a half circle. If $s \subset \partial T_{j}$ then type $(r)=1$. Otherwise, type $(r)=2$.

Induction precondition: Let the reachable regions of $\mathcal{R} \mathcal{R}^{1}\left(S, T_{j}\right), \ldots, \mathcal{R}^{i-1}\left(S, T_{j}\right)$ be either of type 1 or of type 2 .

$i-1 \Rightarrow i$ :

$r^{\prime} \subset T_{j}$ : The $c$-oriented reachable region of $r^{\prime}$ in $T_{j}$ clearly is of type 1.

$\overline{r^{\prime} \subset T_{k}},(k<j-1)$ or $\left(k=j-1\right.$ and $\left.r^{\prime} \cap C_{j}=\emptyset\right)$ : Analogously to case $i=1, j>1$. $\left.\overline{r^{\prime} \subset T_{j-1}, r^{\prime} \cap C_{j} \neq \emptyset \text { : Let } r^{+}=\left\{q \in T_{j} \mid \exists q^{\prime} \in r^{\prime} \backslash\right.} C_{j}, \lambda>0, q^{\prime}+\lambda c=q\right\}$ and $r^{-}=\{q \in$ $\left.\overline{T_{j} \mid \exists q^{\prime} \in r^{\prime} \backslash C_{j}, \lambda<0}, q^{\prime}+\lambda c=q\right\}$. Clearly, $r=r^{+} \cup r^{-}$. Let $s^{+}=\left(\partial C_{j} \backslash \partial T_{j-1}\right) \cap r^{+}$and $s^{-}=\left(\partial C_{j} \backslash \partial T_{j-1}\right) \cap r^{-}$.

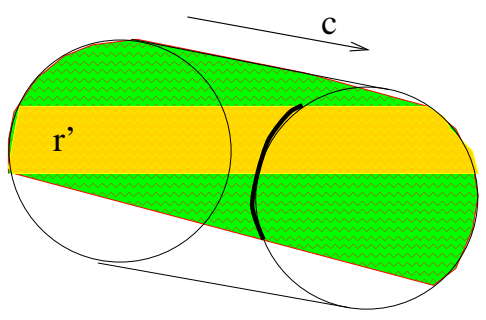

Figure 10: $r^{\prime}$ (shaded light) generates one connected boundary segment $s^{+}$(solid) for the forward extension in orientation $c$.

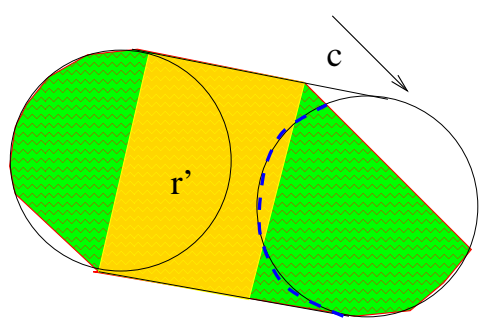

Figure 11: $r^{\prime}$ generates one connected boundary segment $s^{+}$(dashed) for the forward extension in orientation $c$.

We clearly now can subdivide $s^{+}$and $s^{-}$into connected boundary segments. For complexity reasons we show that we yield altogether at most two connected boundary segments. That is, if $s^{+}\left(s^{-}\right)$contains two disjoint segments then $s^{-}=\emptyset$ $\left(s^{+}=\emptyset\right)$. Figures 10-13 show the possibilities of a region of type 1 to intersect $C_{j}$. Note that a region in $T_{j-1}$ of type 2 has empty intersection with $C_{j}$. In the first two cases we yield one connected boundary segment (see Figure 10,11). In the third case depending on $c$ we either yield two boundary segments in $s^{+}$and $s^{-}=\emptyset$ (see Figure 13) or two boundary segments in $s^{-}$ and $s^{+}=\emptyset$ or one in $s^{+}$and one in $s^{-}$(see Figure 12).

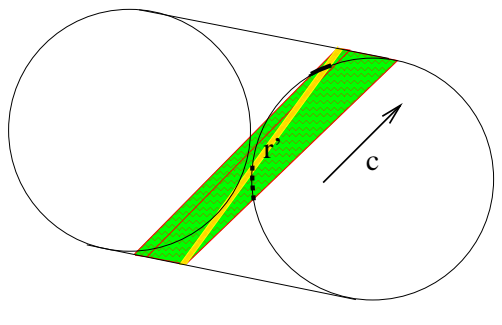

Figure 12: $r^{\prime}$ generates one connected boundary segment $s^{+}$ (dashed) and one boundary segment $s^{-}$(solid) for the forward extension in orientation $c$.

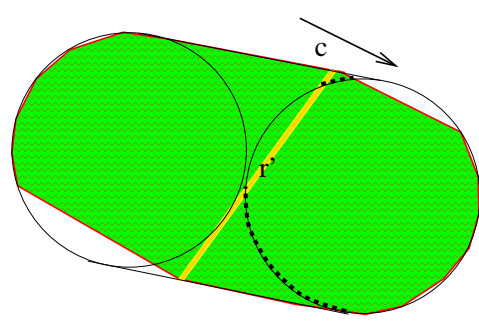

Figure 13: $r^{\prime}$ generates two connected boundary segment $s^{+}$(dashed) for the forward extension in orientation $c$.

In the next two observations we show how to shrink the number of reachable regions by deleting redundant reachable regions and unifying reachable regions.

Observation 4.3 Let $r$ be a c-oriented reachable region in $\mathcal{R} \mathcal{R}^{i}\left(S, T_{j}, c\right)$. Then, $r$ can be deleted in case that there exists a $r^{\prime} \in \mathcal{R} \mathcal{R}^{i}\left(S, T_{j}, c\right)$ with $r \subseteq r^{\prime}$. 
Observation 4.4 Let $r$ and $r^{\prime}$ be two c-oriented reachable regions in $\mathcal{R} \mathcal{R}^{i}\left(S, T_{j}, c\right)$ with $r \cap r^{\prime} \neq$ $\emptyset, r \nsubseteq r^{\prime}$, and $r^{\prime} \nsubseteq r$. Let $r$ and $r^{\prime}$ be reachable regions of type 1 or of type 2 with equal $\tau$. We can unify $r$ and $r^{\prime}$ for the computation of the reachable region of $r$ and $r^{\prime}$.

Note that we must store a back pointer from $r \cup r^{\prime}$ to $r$ and $r^{\prime}$ since during the computation of the link path we have to follow the links backwards until we reach the start set $S$. The classification of a reachable region in type 1 and type 2 leads to the notation that $\mathcal{R} \mathcal{R}^{i}\left(S, T_{j}, c\right)$ consists of a set of reachable regions and a reachable region is an element of $\mathcal{R} \mathcal{R}^{i}\left(S, T_{j}, c\right)$.

Let $\bar{c}$ be the orthogonal orientation to $c$. The projection of a tube $T_{j}$ to $\bar{c}$ yields an interval $I\left(T_{j}, c\right)$.

Observation 4.5 Each c-oriented reachable region in $T_{j}$ is uniquely represented by an interval in $I\left(T_{j}, c\right)$ (namely the projection of the reachable region to $\bar{c}$ ) and a tag specifying whether the reachable region is of type 1 or type 2 accompanied by the orientation $\tau$.

In a further tag field a reference to the generating reachable region is stored. This property we use to store the reachable regions efficiently. We associate three sets of intervals with each $\mathcal{R R}^{i}\left(S, T_{j}, c\right): \mathcal{I}_{i}\left(S, T_{j}, c, \pm\right)$ contains all intervals corresponding to reachable regions of type 1. $\mathcal{I}_{i}\left(S, T_{j}, c,+\right)$ contains all intervals corresponding to reachable regions of type 2 with $\tau=+c$ and $\mathcal{I}_{i}\left(S, T_{j}, c,-\right)$ with $\tau=-c$.

Procedure 1 Insertion ( $c$-oriented reachable region $r, \mathcal{R R}^{i}\left(S, T_{j}, c\right)$ )

1) $r_{p}:=$ the projection of $r$ onto $\bar{c}$;

2) if type $(r)=1$ then \{

3) if $\exists r_{p}^{\prime} \in \mathcal{I}_{i}\left(S, T_{j}, c, \pm\right)$ such that $r_{p} \subseteq r_{p}^{\prime}$ then

4) return;

5) else \{

6) for all $r_{p}^{\prime} \in \mathcal{I}_{i}\left(S, T_{j}, c, \pm\right) \cup \mathcal{I}_{i}\left(S, T_{j}, c,+\right) \cup \mathcal{I}_{i}\left(S, T_{j}, c,-\right)$ such that $r_{p}^{\prime} \subset r_{p}$ do

7) delete $r_{p}^{\prime}$ from the structure;

8) insert $r$ into $\mathcal{I}_{i}\left(S, T_{j}, c, \pm\right)$;

9) $\} \quad\}$

10) else \{

11) if $\exists r_{p}^{\prime} \in \mathcal{I}_{i}\left(S, T_{j}, c, \pm\right) \cup \mathcal{I}_{i}\left(S, T_{j}, c,+\right) \cup \mathcal{I}_{i}\left(S, T_{j}, c,-\right)$ such that $r_{p}^{\prime} \subseteq r_{p}$ then

12) return;

13) else \{

14) if type $(r)=2$ with $\tau=+c$ then \{

15) for all $r_{p}^{\prime} \in \mathcal{I}_{i}\left(S, T_{j}, c,+\right)$ such that $r_{p}^{\prime} \subset r_{p}$ do

16) delete $r_{p}^{\prime}$ from the structure;

17) insert $r$ into $\mathcal{I}_{i}\left(S, T_{j}, c,+\right)$;

18) $\}$

19) else \{

20) for all $r_{p}^{\prime} \in \mathcal{I}_{i}\left(S, T_{j}, c,-\right)$ such that $r_{p}^{\prime} \subset r_{p}$ do

21) delete $r_{p}^{\prime}$ from the structure;

22) insert $r$ into $\mathcal{I}_{i}\left(S, T_{j}, c,-\right)$;

23) $\} \quad\} \quad\}$ 
1) for each $x \in\{ \pm,+,-\}$ do \{

2) let $r_{1}, \ldots, r_{l}$ be the intervals of $\mathcal{I}_{i}\left(S, T_{j}, c, x\right)$;

3) compute the minimum set of intervals $r_{1}^{\star}, \ldots, r_{k}^{\star}$ such that $\bigcup_{e=1}^{l} r_{e}=\bigcup_{f=1}^{k} r_{f}^{\star}$;

4) with each $r_{f}^{\star}$ we associate all $r_{e}$ with $r_{f}^{\star} \cap r_{e} \neq \emptyset$;

5) Insert $r_{1}^{\star}, \ldots, r_{k}^{\star}$ into $\mathcal{I}_{i}^{\prime}\left(S, T_{j}, c, x\right)$;

6) \}

6) for all $r_{f} \in \mathcal{I}_{i}^{\prime}\left(S, T_{j}, c,+\right) \cup \mathcal{I}_{i}^{\prime}\left(S, T_{j}, c,-\right)$ such that $r_{f} \subset \mathcal{I}_{i}^{\prime}\left(S, T_{j}, c, \pm\right)$ do

7) delete $r_{f}$ from the data structure;

8) return $\mathcal{I}_{i}^{\prime}\left(S, T_{j}, c, \pm\right), \mathcal{I}_{i}^{\prime}\left(S, T_{j}, c,+\right), \mathcal{I}_{i}^{\prime}\left(S, T_{j}, c,-\right)$;

We insert a $c$-oriented reachable region $r$ into $\mathcal{R} \mathcal{R}^{i}\left(S, T_{j}, c\right)$ only if it is not yet contained in $\mathcal{R} \mathcal{R}^{i}\left(S, T_{j}, c\right)$. In this case we delete all $c$-oriented reachable regions $r^{\prime}$ that are contained in $r$ see Observation 4.3, and Procedure 1 . Before we compute $\mathcal{R} \mathcal{R}^{i+1}\left(S, T_{j}\right)$ we unify the reachable regions in $\mathcal{R R}^{i}\left(S, T_{j}, c\right)$ for each $c \in \mathcal{C}$ in order to shrink their number (see Observation 4.4 , and Procedure 2). Let $x \in\{ \pm,+,-\}$. The insertion operations in $\mathcal{I}_{i}\left(S, T_{j}, c, x\right)$ have the property that each two intervals $i_{1}$ and $i_{2}$ in $\mathcal{I}_{i}\left(S, T_{j}, c, x\right)$ are either disjoint or $i_{1} \nsubseteq i_{2}$ and $i_{1} \nsupseteq i_{2}$. We suggest handling each $\mathcal{I}_{i}\left(S, T_{j}, c, x\right)$ as an ordered list. Thus, the insertion, deletion, and search for an interval can be done in $\mathcal{O}(\log l)$ time, when $l$ is the length of the list. Let $u$ be an interval that contains $k$ intervals of the list. Since these $k$ intervals are consecutive in the list, the deletion or search of these intervals can be done in $\mathcal{O}(\log l+k)$ time.

If we once have reached a point $p \in T_{j}$ we can estimate the maximum number of iterations that we have to compute extensions into that tube.

Observation 4.6 Let $p \in \mathcal{R R}^{i}\left(S, T_{j}\right)$. Then, $C_{j} \subset \mathcal{R} \mathcal{R}^{i+3}\left(S, T_{j}, c\right)$, for each $c \in \mathcal{C}$.

Proof: Since $p \in \mathcal{R R}^{i}\left(S, T_{j}\right)$, it follows that there exists a $p^{\prime} \in C_{j}$ with $p^{\prime} \in \mathcal{R}^{i}\left(S, T_{j}\right)$. Each point $q \in C_{j}$ is reachable from $p^{\prime}$ with at most two links. Since for each two clockwise next orientations $c, \dot{c} \in \mathcal{C}$ the angle $\varangle(c, \dot{c}) \leq \frac{\pi}{2}, C_{j}$ is covered with reachable regions in the second iteration and thus with a $c$-oriented reachable region in the third iteration.

This ends our speed-up investigations. Recapitulating, we compute the reachable regions $\mathcal{R}^{i}\left(S, T_{j}\right)$ by using dynamic programming, together with the interval lists as data structure, the insertion and update technique, and the sensitive computation of reachable regions. That is, we compute a $c$-oriented reachable region in a tube only in case when the tube is not yet covered with $c$-oriented reachable regions. Furthermore, we compute a forward extension into a tube $T_{j}$ only in case when the circle $C_{j}$ is not yet covered with reachable regions. (See Algorithm 2.)

\section{$5 \quad$ Runtime and Space Calculation}

We now calculate the maximum number of reachable regions in each tube. According to our Recursion Formula 3.7 we will estimate the number of $c$-oriented reachable regions in a tube $T$ that is generated by

1. non $c$-oriented reachable regions in $T$ (see Lemma 5.1). 


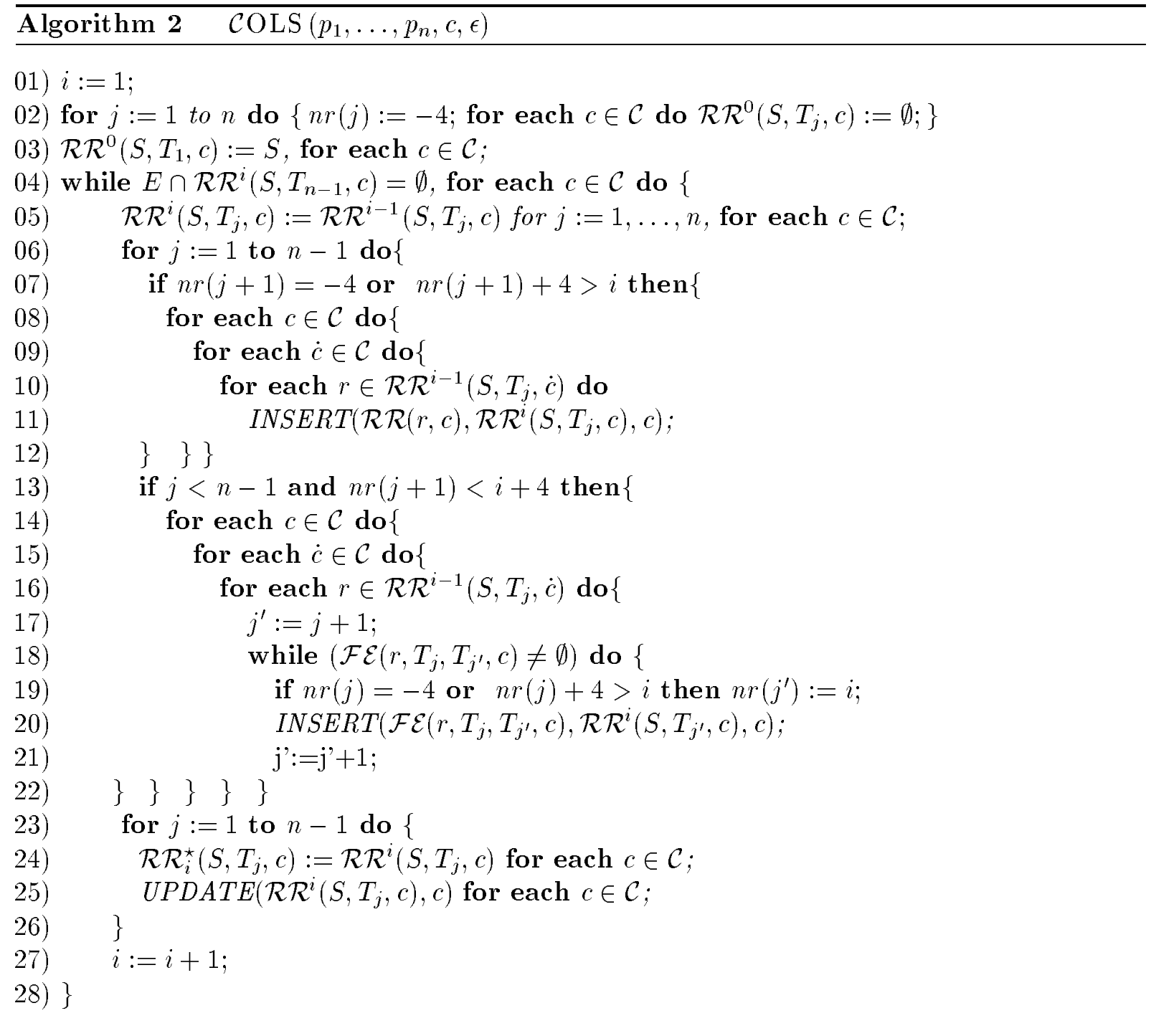


2. forward extensions of non $c$-oriented reachable regions into $T$ (see Corollary 5.2).

3. c-oriented reachable regions in $T$ (see Observation 5.3).

4. forward extensions of $c$-oriented reachable regions into $T$ (see Lemma 5.4).

With these calculations we are able to estimate the maximum number of reachable regions in $\mathcal{R}^{i}\left(S, T_{j}, c\right)$ (see Theorem 5.5) which leads to the runtime and space of the algorithm.

We call two reachable regions $r_{1}, r_{2}$ of one orientation in a tube disjoint, if $r_{1} \cap r_{2}=$ $\emptyset$, type $\left(r_{1}\right) \neq \operatorname{type}\left(r_{2}\right)$, or type $\left(r_{1}\right)=\operatorname{type}\left(r_{2}\right)=2$ and $\tau\left(r_{1}\right) \neq \tau\left(r_{2}\right)$. We call the Update Procedure 2 with $\mathcal{R} \mathcal{R}^{i}\left(S, T_{j}, c\right)$ before computing the sets $\mathcal{R} \mathcal{R}^{i+1}\left(S, T_{j}, c\right)$, for $j=1, \ldots, n-$ 1. Therefore, we only have to take into account the number of disjoint reachable regions in $\mathcal{R R}^{i}\left(S, T_{j}, c\right)$.

Lemma 5.1 Let $r_{1}, \ldots, r_{k}$ be the c-oriented reachable regions in $\mathcal{R} \mathcal{R}^{i}\left(S, T_{j}, c\right)$. Let the angle between $c$ and $\dot{c}$ be $\alpha . \quad r_{1}, \ldots, r_{k}$ create at most $\left\lceil\frac{\pi}{2 \alpha}\right\rceil$ disjoint $\dot{c}$-oriented reachable regions in $\mathcal{R}^{i+1}\left(S, T_{j}, \dot{c}\right)$.

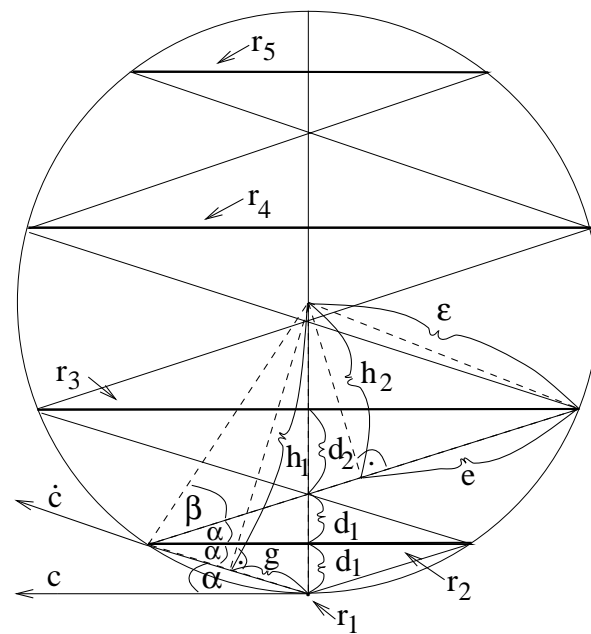

Figure 14: By knowing $C_{j}, \epsilon$, and $\alpha$ we can successively compute $\beta, d_{1}$, $d_{2}, \ldots, g, h_{1}, h_{2}, \ldots, e, \ldots$
Proof: From Theorem 4.2 we know that the reachable regions can be categorized into 2 types. Each reachable region $r_{a} \cap C_{j}$ is defined by the inner of two parallel $c$ oriented lines cut with $C_{j}, 1 \leq a \leq k$. The $\dot{c}$-oriented reachable region of $r_{a}$ and $r_{b}$ can only be disjoint if the $\dot{c}$-oriented reachable region of $r_{a} \cap C_{j}$ and $r_{b} \cap C_{j}$ are disjoint, $1 \leq a, b \leq k$. We therefore get an upper bound on the number of disjoint $\dot{c}$-oriented reachable regions of $r_{1}, \ldots, r_{k}$ when we compute the number of disjoint $\dot{c}$-oriented reachable regions of $r_{1}^{\prime}=r_{1} \cap C_{j}, \ldots, r_{k}^{\prime}=$ $r_{k} \cap C_{k}$. Now, let $r_{a} \subset r_{b}$. Since $\mathcal{R} \mathcal{R}\left(r_{a}, \dot{c}\right) \subset \mathcal{R} \mathcal{R}\left(r_{b}, \dot{c}\right)$, we can assume that $r_{1}^{\prime}, \ldots, r_{k}^{\prime}$ have minimum diameter and are disjoint. We further assume that $r_{1}^{\prime}, \ldots, r_{k}^{\prime}$ have minimum distance to each other and are ordered according to orientation $\bar{c}$. Therefore, $r_{1}^{\prime}$ has distance $\epsilon-\delta$ to the center of $C_{j}$ where $\delta$ is a number approaching 0 . For convenience we say that $r_{1}^{\prime}$ has distance $\epsilon$ to the center of $C_{j}$. Please confer Figure 14 for the following considerations. Trigonometric calculations yield that $r_{2}^{\prime}$ must have distance greater than $d_{1}=2 \epsilon \sin ^{2}(\alpha)$ to $r_{1}^{\prime}$, otherwise the $\dot{c}$-oriented reachable regions of $r_{1}^{\prime}$ and $r_{2}^{\prime}$ would intersect. In case that $d_{1}>\epsilon$ we know that at most 2 disjoint $\dot{c}$-oriented reachable regions can be created. Similar computation yields that $r_{3}^{\prime}$ must have distance greater than $d_{2}+d_{1}=2 \epsilon \sin (\alpha) \sin (3 \alpha)$ to $r_{2}^{\prime}$ or distance greater than $d_{2}+2 d_{1}$ to $r_{1}^{\prime}$. In general $r_{i}^{\prime}$ has to have distance greater than $d_{i-1}+d_{i-2}$ to $r_{i-1}^{\prime}$ or distance greater than $\sum_{j=1}^{i-2} 2 d_{j}+d_{i-1}$ to $r_{1}^{\prime}$, with

$$
d_{i}= \begin{cases}2 \epsilon \sin (\alpha) \sum_{j=1}^{i}(-1)^{j} \sin ((2 j-1) \alpha) & \text { if } i \text { is even, } \\ 2 \epsilon \sin (\alpha) \sum_{j=1}^{i}(-1)^{j+1} \sin ((2 j-1) \alpha) & \text { otherwise. }\end{cases}
$$

These equations are only valid while $r_{i}^{\prime}$ has distance smaller than $\epsilon$ to $r_{1}^{\prime}$ which is the case for $i<\frac{\pi}{4 \alpha}-\frac{1}{2}$. It follows that at most $\left\lceil\frac{\pi}{4 \alpha}\right\rceil$ disjoint $\dot{c}$-oriented reachable regions can be created from the $c$-oriented reachable regions with distance at most $\epsilon$ to $r_{1}$ (including $r_{1}$ ). Thus at most $\left\lceil\frac{\pi}{2 \alpha}\right\rceil$ disjoint $\dot{c}$-oriented reachable regions can be created from $r_{1}, \ldots, r_{k}$. 
Corollary 5.2 A maximum of $3\left\lceil\frac{\pi}{2 \alpha_{\min }}\right\rceil(|\mathcal{C}|-1)$ disjoint c-oriented reachable regions can exist in $\mathcal{R} \mathcal{R}\left(\mathcal{R} \mathcal{R}^{i}\left(S, T_{j}\right) \backslash \mathcal{R} \mathcal{R}^{i}\left(S, T_{j}, c\right), c\right) \bigcup \bigcup_{k=1}^{j-1} \mathcal{F} \mathcal{E}\left(\left(\mathcal{R R}^{i}\left(S, T_{k}\right) \backslash \mathcal{R} \mathcal{R}^{i}\left(S, T_{k}, c\right)\right), T_{k}, T_{j}, c\right)$,

Observation 5.3 1. Each $\mathcal{R R}^{i}\left(S, T_{1}, c\right)$ contains one unique reachable region, $c \in \mathcal{C}$.

2. Let $r \in \mathcal{R}^{i}\left(S, T_{j}, c\right)$ with type $(r)=1$. Then, $\mathcal{R} \mathcal{R}(r, c)=r$.

3. Let $r \in \mathcal{R R}^{i}\left(S, T_{j}, c\right)$ with type $(r)=2$ and $\tau \in\{+c,-c\}$. Then, $\mathcal{R} \mathcal{R}(r, c) \supset r$ and type $(\mathcal{R} \mathcal{R}(r, c))=1$.

In the next lemma we show that in case that a $c$-oriented reachable region has a $c$-oriented forward extension from tube $T_{k}$ to tube $T_{j}$, then at most one $c$-oriented reachable region in each tube $T_{k+1}, \ldots, T_{j-1}$ has a forward extension into $T_{j}$.

Lemma 5.4 Let $\mathcal{F} \mathcal{E}\left(\mathcal{R} \mathcal{R}^{i}\left(S, T_{k}, c\right), T_{k}, T_{j}, c\right) \neq \emptyset, k<j<n$. Then, $\mathcal{F} \mathcal{E}\left(\mathcal{R} \mathcal{R}^{i}\left(S, T_{l}, c\right), T_{l}, T_{j}, c\right)$ contains at most one reachable region for any $l, k<l<j$.

Proof: Firstly, we study some special properties of a $c$-oriented reachable region that is extended with orientation $c$. Let $r^{\prime} \in \mathcal{R} \mathcal{R}^{i}\left(S, T_{k}, c\right)$ such that $r=\mathcal{F} \mathcal{E}\left(r^{\prime}, T_{k}, T_{j}, c\right) \neq \emptyset$. Then, type $\left(r^{\prime}\right)=2$ with $\tau\left(r^{\prime}\right) \in\{c,-c\}$. From Theorem 4.2, it follows that there exists a $s^{\prime} \subset \partial C_{k}$ which is the $r^{\prime}$ defining boundary segment. The definition of a forward extension yields that for all $u \in r^{\prime}$ there exists a $v \in s^{\prime}$ and a $\lambda>0$ such that $u=\lambda \tau\left(r^{\prime}\right)$. Since $r^{\prime}$ can only be extended in direction $-\tau\left(r^{\prime}\right)$ we get $\tau(r)=-\tau\left(r^{\prime}\right)$. Furthermore, for all $u \in r$ there exists a $v \in s$ and a $\lambda>0$ such that $u=v-\lambda \tau\left(r^{\prime}\right)$.

Secondly, under the assumption that $\mathcal{F} \mathcal{E}\left(\mathcal{R} \mathcal{R}^{i}\left(S, T_{k}, c\right), T_{k}, T_{j}, c\right) \quad \neq \quad \emptyset$ and $\mathcal{F E}\left(\mathcal{R} \mathcal{R}^{i}\left(S, T_{l}, c\right), T_{l}, T_{j}, c\right) \neq \emptyset, k<l<j, r_{l}^{\prime} \subseteq \mathcal{R} \mathcal{R}^{i}\left(S, T_{l}, c\right)$ with $\mathcal{F} \mathcal{E}\left(r_{l}^{\prime}, T_{l}, T_{j}, c\right) \neq \emptyset$ and any $r_{l}^{\prime \prime} \subseteq \mathcal{R} \mathcal{R}^{i-1}\left(S, T_{m}, c\right)$ with $\mathcal{F E}\left(r_{l}^{\prime \prime}, T_{m}, T_{l}, c\right) \neq \emptyset$ it follows that $r_{l}^{\prime} \cap \mathcal{F E}\left(r_{l}^{\prime \prime}, T_{m}, T_{l}, c\right)=\emptyset$, for any $m<l$ : Let $r_{k}^{\prime} \in \mathcal{R} \mathcal{R}^{i}\left(S, T_{k}, c\right)$ such that $r_{k}=\mathcal{F} \mathcal{E}\left(r_{k}^{\prime}, T_{k}, T_{j}, c\right) \neq \emptyset$ and let $r_{l}^{\prime} \in \mathcal{R} \mathcal{R}^{i}\left(S, T_{l}, c\right)$ such that $r_{l}=\mathcal{F} \mathcal{E}\left(r_{l}^{\prime}, T_{l}, T_{j}, c\right) \neq \emptyset$, with $k<l<j$. It follows that $\tau\left(r_{k}^{\prime}\right)=-\tau\left(r_{k}\right)$ and $\tau\left(r_{l}^{\prime}\right)=-\tau\left(r_{l}\right)$. Let $s_{k}^{\prime}$ (resp. $s_{l}^{\prime}$ ) be the $r_{k}^{\prime}$ (resp. $r_{l}^{\prime}$ ) defining boundary segment. Then, there exists an $u \in s_{k}^{\prime}$ such that $u+\lambda \tau\left(r_{k}\right)$ intersects $C_{k}, \ldots, C_{j}$ in this order. Analogously, there exists an $v \in s_{l}^{\prime}$ such that $v+\lambda \tau\left(r_{l}\right)$ intersects $C_{l}, \ldots, C_{j}$ in this order. Since $k<l<j$ and $C_{j} \cap C_{j+1}=\emptyset$, for $1 \leq j \leq n-1$, it follows that $\tau\left(r_{k}\right)=\tau\left(r_{l}\right)$.

We now assume that there exists a reachable region $r_{l}^{\prime \prime} \in \mathcal{R} \mathcal{R}^{i-1}\left(S, T_{m}, c\right)$ such that $r_{l}^{\prime} \supseteq$ $\mathcal{F E}\left(r_{l}^{\prime \prime}, T_{m}, T_{l}, c\right)$. It follows that type $\left(r_{l}^{\prime \prime}\right)=2$ with $\tau\left(r_{l}^{\prime \prime}\right)=-\tau\left(r_{l}^{\prime}\right)$. Let $s_{l}^{\prime \prime}$ be the $r_{l}^{\prime \prime}$ defining boundary segment. Then, $s_{l}^{\prime \prime} \subset \partial C_{m}$ and there exists a $v \in s_{l}^{\prime \prime}$ such that $v+\lambda \tau\left(r_{l}^{\prime}\right)$ intersects $C_{m}, \ldots, C_{l}$ in this order. Since there exists an $u \in s_{k}^{\prime}$ such that $u+\lambda \tau\left(r_{k}\right)$ intersects $C_{k}, \ldots, C_{j}$ in this order, $\tau\left(r_{k}\right)=-\tau\left(r_{l}^{\prime}\right), m, k<l$, and $C_{l-1} \cap C_{l}=\emptyset$ we come to a contradiction.

Thirdly, we assume that $\mathcal{F} \mathcal{E}\left(\mathcal{R} \mathcal{R}^{i}\left(S, T_{l}, c\right), T_{l}, T_{j}, c\right)$ contains 2 disjoint reachable regions and lead this to a contradiction: Let $r_{l_{1}}^{\prime} \in \mathcal{R} \mathcal{R}^{i}\left(S, T_{l}, c\right)$ such that $r_{l_{1}}=\mathcal{F} \mathcal{E}\left(r_{l_{1}}^{\prime}, T_{l}, T_{j}, c\right) \neq \emptyset$ and let $r_{l_{2}}^{\prime} \in \mathcal{R R}^{i}\left(S, T_{l}, c\right)$ such that $r_{l_{2}}=\mathcal{F} \mathcal{E}\left(r_{l_{2}}^{\prime}, T_{l}, T_{j}, c\right) \neq \emptyset$. We show that $r_{l_{1}} \cap r_{l_{2}} \neq \emptyset$ : From the first case it follows that $\tau\left(r_{l_{1}}^{\prime}\right)=\tau\left(r_{l_{2}}^{\prime}\right)=\tau\left(r_{k}^{\prime}\right)=-\tau\left(r_{l_{1}}\right)=-\tau\left(r_{l_{2}}\right)=-\tau\left(r_{k}\right)$. Hence, there exists a reachable region $r_{l_{1}}^{\prime \prime} \in \mathcal{R} \mathcal{R}^{i-1}\left(S, T_{m_{1}}, c\right)$ such that $r_{l_{1}}^{\prime} \supseteq \mathcal{F E}\left(r_{l_{1}}^{\prime \prime}, T_{m_{1}}, T_{l}, c\right)$ and $r_{l,}^{\prime \prime} \in \mathcal{R R}^{i-1}\left(S, T_{m_{2}}, c\right)$ such that $r_{l_{2}}^{\prime} \supseteq \mathcal{F E}\left(r_{l_{2}}^{\prime \prime}, T_{m_{2}}, T_{l}, c\right), m_{1}, m_{2}<l$.

Claim: $m_{1}=m_{2}=l-1$ : Suppose $m_{1}<l-1$. Now, there exists a $v \in r_{l_{1}}^{\prime \prime}$ such that $v+\lambda \tau\left(r_{l_{1}}^{\prime}\right)$ intersects $C_{m_{1}+1}, \ldots, C_{l}$ in this order. Since there exists an $u \in s_{k}^{\prime}$ such that $u+\lambda \tau\left(r_{k}\right)$ intersects $C_{k}, \ldots, C_{j}$ in this order, $\tau\left(r_{k}\right)=-\tau\left(r_{l_{1}}^{\prime}\right), m_{1}, k<l-1$, and $C_{l-1} \cap C_{l}=\emptyset$ we come to a contradiction. 
Let $X$ be an arbitrary region in the plane. Let $I(X)$ be the interval when projecting $X$ onto $\bar{c}$. By Lemma 4.1 follows that $r_{l_{1}}^{\prime \prime} \cap C_{l-1} \neq \emptyset$ and $r_{l_{2}}^{\prime \prime} \cap C_{l-1} \neq \emptyset$. Therefore, $I\left(r_{l_{1}}^{\prime \prime}\right) \cap$ $I\left(C_{l-1}\right) \neq \emptyset$ and $I\left(r_{l_{2}}^{\prime \prime}\right) \cap I\left(C_{l-1}\right) \neq \emptyset$. Furthermore, since $r_{l_{1}}^{\prime} \supseteq \mathcal{F E}\left(r_{l_{1}}^{\prime \prime}, T_{l-1}, T_{l}, c\right) \neq \emptyset$ and $r_{l_{2}}^{\prime} \supseteq \mathcal{F} \mathcal{E}\left(r_{l_{2}}^{\prime \prime}, T_{l-1}, T_{l}, c\right) \neq \emptyset$ we get that $I\left(r_{l_{1}}^{\prime \prime}\right) \cap I\left(C_{l}\right) \neq \emptyset$ and $I\left(r_{l_{2}}^{\prime \prime}\right) \cap I\left(C_{l}\right) \neq \emptyset$. A necessary condition for $r_{l_{1}} \cap r_{l_{2}}=\emptyset$ is that $I\left(r_{l_{1}}^{\prime \prime}\right) \cap I\left(r_{l_{2}}^{\prime \prime}\right)=\emptyset$. This is only possible if $I\left(r_{l_{1}}^{\prime \prime}\right) \subseteq I\left(C_{l-1}\right) \cap I\left(C_{l}\right)$ and $I\left(r_{l_{2}}^{\prime \prime}\right) \subseteq I\left(C_{l-1}\right) \cap I\left(C_{l}\right)$. But then, there exists a $v \in r_{l_{1}}^{\prime \prime} \cap C_{l-1}$ such that $v+\lambda \tau\left(r_{l_{1}}^{\prime}\right)$ intersects $C_{l-1}, C_{l}$ in this order. Since there exists an $u \in s_{k}^{\prime}$ such that $u+\lambda \tau\left(r_{k}\right)$ intersects $C_{k}, \ldots, C_{j}$ in this order, $\tau\left(r_{k}\right)=-\tau\left(r_{l_{1}}^{\prime}\right), k<l-1$, and $C_{l-1} \cap C_{l}=\emptyset$ we come to a contradiction here.

Theorem 5.5 $\mathcal{R R}^{i}\left(S, T_{j}, c\right)$ contains at most $3 j\left\lceil\frac{\pi}{2 \alpha_{\min }}\right\rceil(|\mathcal{C}|-1)$ disjoint reachable regions.

Proof: The number of reachable regions in $\mathcal{R} \mathcal{R}^{i}\left(S, T_{j}, c\right)$ is the sum of the reachable regions in:

(1) $\mathcal{R} \mathcal{R}\left(\mathcal{R} \mathcal{R}^{i}\left(S, T_{j}\right) \backslash \mathcal{R} \mathcal{R}^{i}\left(S, T_{j}, c\right), c\right)$,

(2) $\bigcup_{k=1}^{j-1} \mathcal{F E}\left(\left(\mathcal{R} \mathcal{R}^{i}\left(S, T_{k}\right) \backslash \mathcal{R} \mathcal{R}^{i}\left(S, T_{k}, c\right)\right), T_{k}, T_{j}, c\right)$,

(3) $\mathcal{R} \mathcal{R}\left(\mathcal{R R}^{i}\left(S, T_{j}, c\right), c\right)$, and (4) $\bigcup_{k=1}^{j-1} \mathcal{F E}\left(\mathcal{R} \mathcal{R}^{i}\left(S, T_{k}, c\right), T_{k}, T_{j}, c\right)$.

(1) and (2): Lemma 5.1 and Corollary 5.2 yield that a maximum of $3\left\lceil\frac{\pi}{2 \alpha_{\min }}\right\rceil(|\mathcal{C}|-1)$ disjoint $c$-oriented reachable regions that were generated by the reachable regions in $\mathcal{R R}^{i}\left(S, T_{j}\right) \backslash \mathcal{R} \mathcal{R}^{i}\left(S, T_{j}, c\right)$ and $\bigcup_{k=1}^{j-1} \mathcal{F} \mathcal{E}\left(\left(\mathcal{R} \mathcal{R}^{i}\left(S, T_{k}\right) \backslash \mathcal{R} \mathcal{R}^{i}\left(S, T_{k}, c\right)\right), T_{k}, T_{j}, c\right)$ can exist in $\mathcal{R R}^{i+1}\left(S, T_{j}, c\right)$.

(3): Observation 5.3 yields that the number of reachable regions in $\mathcal{R} \mathcal{R}\left(\mathcal{R} \mathcal{R}^{i}\left(S, T_{j}, c\right), c\right)$ is at most as big as the number of reachable regions in $\mathcal{R} \mathcal{R}^{i}\left(S, T_{j}, c\right)$.

(4): Lemma 5.4 yields that the number of reachable regions in $\bigcup_{k=1}^{j-1} \mathcal{F} \mathcal{E}\left(\mathcal{R} \mathcal{R}^{i}\left(S, T_{k}, c\right), T_{k}, T_{j}, c\right)$ is smaller or equal to the maximum number of $c$-oriented reachable regions in $\mathcal{R} \mathcal{R}^{i}\left(S, T_{k}, c\right)$ plus $j-k-1$.

By induction on $j$ we show that the number of reachable regions in $\mathcal{R} \mathcal{R}^{i}\left(S, T_{j}, c\right)$ is bounded by $3 j\left\lceil\frac{\pi}{2 \alpha_{\min }}\right\rceil(|\mathcal{C}|-1)$ :

$\mathrm{j}=1$ : Observation 5.3 yields that the number of reachable regions in $\mathcal{R}^{i}\left(S, T_{1}, c\right)$ is equal to $1 \leq 3\left\lceil\frac{\pi}{2 \alpha_{\min }}\right\rceil(|\mathcal{C}|-1)$.

Induction precondition: Let the condition be true for $1, \ldots, j-1$.

$\overline{j-1 \Rightarrow j:}$ The number of reachable regions in $\mathcal{R R}^{i}\left(S, T_{j}, c\right)$ is bounded by the number of reachable regions in $\mathcal{R} \mathcal{R}\left(\mathcal{R} \mathcal{R}^{i}\left(S, T_{j}\right) \backslash \mathcal{R} \mathcal{R}^{i}\left(S, T_{j}, c\right), c\right)$, in $\bigcup_{k=1}^{j-1} \mathcal{F E}\left(\left(\mathcal{R R}^{i}\left(S, T_{k}\right) \backslash \mathcal{R} \mathcal{R}^{i}\left(S, T_{k}, c\right)\right), T_{k}, T_{j}, c\right)$ and the maximum number of $c$-oriented reachable regions in $\mathcal{R}^{i}\left(S, T_{k}, c\right)$ plus $j-k-1$. Applying the induction precondition we yield in total at most $3\left\lceil\frac{\pi}{2 \alpha_{\min }}\right\rceil(|\mathcal{C}|-1)+3(j-1)\left\lceil\frac{\pi}{2 \alpha_{\min }}\right\rceil(|\mathcal{C}|-1)=3 j\left\lceil\frac{\pi}{2 \alpha_{\min }}\right\rceil(|\mathcal{C}|-1)$ disjoint reachable regions.

The estimation of the number of reachable regions leads to the running time and space needed by the algorithm.

Theorem 5.6 Let $L_{\max }=\max _{j=2, \ldots, n}\left\{\frac{\left\|p_{j}-p_{j-1}\right\|_{2}}{2 \epsilon}\right\}+4$. The running time of the algorithm is bounded by $\mathcal{O}\left(n^{2}|\mathcal{C}|^{2} L_{\max }\left\lceil\frac{\pi}{2 \alpha_{\min }}\right\rceil \log \left(n|\mathcal{C}|\left\lceil\frac{\pi}{2 \alpha_{\min }}\right)\right)\right.$. Expressed in the minimum number of links $k$ of $\mathcal{C} O L S$ the algorithm needs $\mathcal{O}\left(k n^{2}|\mathcal{C}|^{2}\left\lceil\frac{\pi}{2 \alpha_{\min }}\right\rceil \log \left(n|\mathcal{C}|\left\lceil\frac{\pi}{2 \alpha_{\min }}\right\rceil\right)\right)$ time.

Proof: Let $L_{j}=\frac{\left\|p_{j+1}-p_{j}\right\|_{2}}{2 \epsilon}+4$. Lemma 3.3 yields that at most $L_{j}$ links are needed to reach each point in $T_{j}$ from a starting set of points in $C_{j}$. Therefore, $L_{\max }$ corresponds to the maximum number of iterations needed to cover any $T_{j}$ with reachable regions, $j=1, \ldots, n$. Theorem 5.5 yields that the number of reachable regions in $\mathcal{R}^{i}\left(S, T_{j}\right)$ is bounded by $|\mathcal{C}| 3 j\left\lceil\frac{\pi}{2 \alpha_{\text {min }}}\right\rceil(|\mathcal{C}|-1)$. 
For each reachable region in $\mathcal{R} \mathcal{R}^{i}\left(S, T_{j}\right),|\mathcal{C}|$ new reachable regions are computed. We assume that the computation of a reachable region in a tube from a reachable region can be done in constant time. Each reachable region is inserted into a list structure. The insertion of all reachable regions into $\mathcal{R} \mathcal{R}^{i}\left(S, T_{j}\right)$ requires at most $\mathcal{O}\left(|\mathcal{C}|^{2} j\left\lceil\frac{\pi}{2 \alpha_{\min }}\right\rceil \log \left(j|\mathcal{C}|\left\lceil\frac{\pi}{2 \alpha_{\min }}\right\rceil\right)\right)$ time. The forward extension of reachable regions into tube $T_{j}$ is processed in four sequencing iterations. Thus, at most $4|\mathcal{C}| 3 j\left\lceil\frac{\pi}{2 \alpha_{\min }}\right\rceil(|\mathcal{C}|-1)$ reachable regions are extended into $T_{j}$. This requires time $\mathcal{O}\left(|\mathcal{C}|^{2} j\left\lceil\frac{\pi}{2 \alpha_{\min }}\right\rceil\right.$ $\left.\log \left(|\mathcal{C}| j\left\lceil\frac{\pi}{2 \alpha_{\min }}\right\rceil\right)\right)$. Altogether, we require at most $\mathcal{O}\left(n L_{\max }\left(n|\mathcal{C}|^{2}\left\lceil\frac{\pi}{2 \alpha_{\min }}\right\rceil \log \left(n|\mathcal{C}|\left\lceil\frac{\pi}{2 \alpha_{\min }}\right\rceil\right)\right)+\right.$ $\mathcal{O}\left(n^{2}|\mathcal{C}|^{2}\left\lceil\frac{\pi}{2 \alpha_{\min }}\right\rceil \log \left(n|\mathcal{C}|\left\lceil\frac{\pi}{2 \alpha_{\min }}\right\rceil\right)\right)=\mathcal{O}\left(n^{2}|\mathcal{C}|^{2} L_{\max }\left\lceil\frac{\pi}{2 \alpha_{\min }}\right\rceil \log \left(n|\mathcal{C}|\left\lceil\frac{\pi}{2 \alpha_{\min }}\right\rceil\right)\right)$ time in the algorithm. Expressed in the minimum number of links $k$ of $\mathcal{C}$ OLS the algorithm requires at most $\mathcal{O}\left(k n^{2}|\mathcal{C}|^{2}\left\lceil\frac{\pi}{2 \alpha_{\min }}\right\rceil \log \left(n|\mathcal{C}|\left\lceil\frac{\pi}{2 \alpha_{\text {min }}}\right\rceil\right)\right)$ time.

Corollary 5.7 The Algorithm needs at most $\mathcal{O}\left(n^{2}|\mathcal{C}|^{2} L_{\max }\left\lceil\frac{\pi}{2 \alpha_{\min }}\right\rceil\right)$ space. Expressed in the minimum number of links $k$ of $\mathcal{C} O L S$ the space is bounded by $\mathcal{O}\left(k n^{2}|\mathcal{C}|^{2}\left\lceil\frac{\pi}{2 \alpha_{\min }}\right\rceil\right)$.

Proof: Each $\mathcal{R} \mathcal{R}^{i}\left(S, T_{j}, c\right)$ needs $\mathcal{O}\left(j|\mathcal{C}|\left\lceil\frac{\pi}{2 \alpha_{\min }}\right\rceil\right)$ space. Thus, $\mathcal{R} \mathcal{R}^{i}\left(S, T_{j}\right)$ needs $\mathcal{O}\left(|\mathcal{C}|^{2} j\left\lceil\frac{\pi}{2 \alpha_{\min }}\right\rceil\right)$ space and $\bigcup_{i=1}^{L_{\max }} \mathcal{R} \mathcal{R}^{i}\left(S, T_{j}\right)$ needs $\mathcal{O}\left(|\mathcal{C}|^{2} j L_{\max }\left\lceil\frac{\pi}{2 \alpha_{\min }}\right\rceil\right)$ space. Thus $\bigcup_{j=1}^{n-1} \bigcup_{i=1}^{I_{\max }} \mathcal{R} \mathcal{R}^{i}\left(S, T_{j}\right)$ needs $\mathcal{O}\left(n^{2}|\mathcal{C}|^{2} L_{\max }\left\lceil\frac{\pi}{2 \alpha_{\min }}\right\rceil\right)$ space. Expressed in the minimum number of links $k$ of $\mathcal{C}$ OLS we have: $\bigcup_{j=1}^{n-1} \mathcal{R} \mathcal{R}^{i}\left(S, T_{j}\right)$ needs $\mathcal{O}\left(n^{2}|\mathcal{C}|^{2}\left\lceil\frac{\pi}{2 \alpha_{\min }}\right\rceil\right)$ space and therefore all reachable regions together need $\mathcal{O}\left(k n^{2}|\mathcal{C}|^{2}\left\lceil\frac{\pi}{2 \alpha_{\min }}\right\rceil\right)$ space.

\section{Relation to the Fréchet Metric}

We note relationships to the Fréchet metric for curve similarity and line simplification [AG95, God91, God98]. Intuitively, two curves $\alpha$ and $\beta$ have Fréchet distance at most $\epsilon$, if a person walking along $\alpha$ can walk a $\operatorname{dog}$ along $\beta$ with a leash of length $\epsilon$. More precisely, two curves have distance at most $\epsilon$ under Fréchet metric if and only if they have monotone parameterizations $\alpha$ and $\beta\left(\alpha, \beta:[0,1] \rightarrow \mathbb{R}^{2}\right)$, such that $\|\alpha(t)-\beta(t)\|_{2} \leq \epsilon$ for all $t \in[0,1]$. Figure 2 shows for each polygonal chain a minimum $\mathcal{C}$-oriented line simplification that has Fréchet distance at most $\epsilon$.

In this section we show that a minimum $\mathcal{C}$-oriented line simplification with Fréchet distance at most $\epsilon$ to $P$ has as many links as a minimum $\mathcal{C}$-oriented line simplification has. Furthermore, we show how to obtain a minimum $\mathcal{C}$-oriented line simplification with Fréchet distance at most $\epsilon$ to $P$ from the reachable regions computed in the algorithm. In the next definition we define the neighborhood of a point $q$ as the connected component of the intersection of $P$ with the $\epsilon$-circle around $q$.

Definition 6.1 (Neighborhood $N(q)$ ) Let $P=\left\langle p_{1}, \ldots, p_{n}\right\rangle$ be a polygonal chain and $Q=$ $\left\langle q_{1}, \ldots q_{m}\right\rangle$ be $a \mathcal{C}$-oriented line approximation of $P$. Let $q$ be a point on $Q, q \in T_{j}, j$ minimal, and $1 \leq j \leq n-1$.

$\underline{q \in T_{j} \backslash C_{j+1}}:$ Let $N(q)=\left\{x \in \overline{p_{j} p_{j+1}}\right.$ such that $\left.\|q-x\|_{2} \leq \epsilon\right\}$.

$\underline{q \in C_{j+1}: \text { Let }} N(q)=\left\{x \in \overline{p_{j} p_{j+1}} \cup \overline{p_{j+1} p_{j+2}}\right.$ such that $\left.\|q-x\|_{2} \leq \epsilon\right\}$.

Let $Q$ be a curve with Fréchet distance at most $\epsilon$ to $P$. From this definition follows that $P$ and $Q$ have monotone parameterizations $\alpha$ and $\beta$ such that $\alpha(t)$ is in the neighborhood of $\beta(t)$. 
Observation 6.2 1. $N(q)$ is a continuous subset of $\overline{p_{j} p_{j+1}}$ or $\left\langle p_{j}, p_{j+1}, p_{j+2}\right\rangle$, respectively.

2. Let $Q$ have Fréchet distance at most $\epsilon$ to $P$. Let $\alpha$ and $\beta$ be monotone parameterizations of $P$ and $Q$, such that $\|\alpha(t)-\beta(t)\|_{2} \leq \epsilon$ for all $t \in[0,1]$. If $\beta(t)=q$ then $\alpha(t) \in N(q)$, $t \in[0,1]$.

We show that a $\mathcal{C}$-oriented line approximation $Q$ of $P$ has Fréchet distance at most $\epsilon$ to $P$ if and only if the neighborhood of $Q$ contains $P$ and for any two points $q^{\prime}$ and $q^{\prime \prime}$ on $Q$ where $q^{\prime}$ precedes $q^{\prime \prime}$ on $Q$ the neighborhood of $q^{\prime \prime}$ does not precede the neighborhood of $q^{\prime \prime}$ on $P$.

Lemma 6.3 Let $P=\left\langle p_{1}, \ldots, p_{n}\right\rangle$ be a polygonal chain and $Q=\left\langle q_{1}, \ldots, q_{m}\right\rangle$ be a $\mathcal{C}$-oriented line approximation of $P$. $Q$ has Fréchet distance at most $\epsilon$ to $P \Leftrightarrow$

(1) for all points $p$ on $P$ there exists a $q$ on $Q$ such that $p \in N(q)$, and

(2) for all points $q^{\prime} \prec q^{\prime \prime}$ on $Q$ we have that $N\left(q^{\prime \prime}\right) \nprec N\left(q^{\prime}\right)$.

Proof: “ $\Rightarrow$ ": (1) is trivially fulfilled. (2) Assume there exists $t<t^{\prime}, t, t^{\prime} \in[0,1]$ with $N\left(\beta\left(t^{\prime}\right)\right) \prec$ $N(\beta(t))$. Let $\alpha(t)$ be the a monotone parameterization of $P$. Since $\alpha(t) \in N(\beta(t))$ and $\alpha\left(t^{\prime}\right) \in$ $N\left(\beta\left(t^{\prime}\right)\right)$ it follows that $\alpha\left(t^{\prime \prime}\right) \prec \alpha\left(t^{\prime}\right)$ - contradiction.

" $\Leftarrow$ :" Let $\alpha^{\prime}$ and $\beta^{\prime}$ be two arbitrary monotone parameterizations of $P$ and $Q$, respectively. Let $F_{\epsilon}=\left\{(s, t) \in[0,1] \times[0,1] \mid\left\|\alpha^{\prime}(s)-\beta^{\prime}(t)\right\|_{2} \leq \epsilon\right\}$. From [AG95] Lemma 4 follows that $P$ and $Q$ have Fréchet distance at most $\epsilon$ if a curve within $F_{\epsilon}$ from $(0,0)$ to $(1,1)$ exists that is monotone in both coordinates. Since $P$ and $Q$ are piecewise linear we know that $N(q) \cap N(q+\delta)=_{\delta \rightarrow 0} N(q)$. Additionally, since $N(q) \neq \emptyset$ and for all points $p$ on $P$ there exists a $q$ on $Q$ such that $p \in N(q)$, it follows that $F_{\epsilon}$ is connected. Since for $t^{\prime}<t^{\prime \prime}, t^{\prime}, t^{\prime \prime} \in[0,1]$ follows that $N\left(\beta^{\prime}\left(t^{\prime \prime}\right)\right) \nprec N\left(\beta^{\prime}\left(t^{\prime}\right)\right)$ it follows the existence of such a curve within $F_{\epsilon}$.

Now, we show that any $\mathcal{C}$-oriented polygonal chain with Fréchet distance at most $\epsilon$ to $P$ is a $\mathcal{C}$-oriented approximation of $P$.

Theorem 6.4 Let $P=\left\langle p_{1}, \ldots, p_{n}\right\rangle$ be a polygonal chain. Let $Q=\left\langle q_{1}, \ldots q_{m}\right\rangle$ be a polygonal chain with $q_{1} \in S, q_{n} \in E$ and $\overrightarrow{q_{i} q_{i+1}} \in \mathcal{C}$. Furthermore, let $Q$ have Fréchet distance at most $\epsilon$ to $P$. Then, $Q$ is a $\mathcal{C}$-oriented approximation of $P$.

Proof: Clearly $q_{i} \in \mathcal{T}, i=1, \ldots, m$. We have to show that the links fulfill the requirements of Problem 2.1. Let $\alpha$ and $\beta$ be two monotone parameterizations of $P$ and $Q$ such that $\|\alpha(t)-\beta(t)\|_{2} \leq \epsilon$ for all $t \in[0,1]$. Let $\overline{q_{i} q_{i+1}}$ be a link of $Q$.

Case $q_{i} \in T_{j}$ and $q_{i+1} \in T_{j}$ : In this case $\overline{q_{i} q_{i+1}}$ lies inside $T_{j}$.

Case $q_{i} \in T_{l}$ and $q_{i+1} \in T_{j}$, such that $l$ is maximal, $j$ is minimal, and $l<j$ : Then, there exists

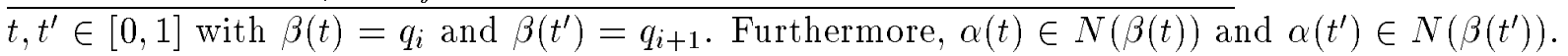
Since $\alpha$ is a monotone parameterization of $P$ there exists $t_{l+1}, \ldots, t_{j}$ with $t \leq t_{l+1}<\cdots<t_{j} \leq t^{\prime}$ and $\alpha\left(t_{k}\right)=p_{k}, k=l+1, \ldots, j$. It follows that $\beta\left(t_{k}\right) \in C_{k}, k=l+1, \ldots, j$. Thus, $\overline{q_{i} q_{i+1}}$ intersects all $C_{l+1}, \ldots, C_{j}$ in this order.

Case $q_{i} \in T_{j}$ and $q_{i+1} \in T_{l}$, such that $l$ is maximal, $j$ is minimal, and $l<j$ : This can not happen since two consecutive $\epsilon$-circles have empty intersection and $Q$ is a Fréchet curve.

After these preparations we are ready to prove our main theorem of this section.

Theorem 6.5 Let $P=\left\langle p_{1}, \ldots, p_{n}\right\rangle$ be a polygonal chain and $Q=\left\langle q_{1}, \ldots, q_{m}\right\rangle$ be a $\mathcal{C}$-oriented line simplification of $P$. Then, there exists a $\mathcal{C}$-oriented line simplification $Q^{\prime}$ with Fréchet distance at most $\epsilon$ to $P$ that has as many links as $Q$ has. 
Proof: From the Backward Extension Lemma 3.6 follows that there exists a $\mathcal{C}$-oriented line simplification $Q$ of $P$ that contains no backward extensions. Let $Q$ be a $\mathcal{C}$-oriented line simplification of $P$ without backward extensions with Fréchet distance greater than $\epsilon$ to $P$. We give a constructive proof that successively transforms $Q$ into a $\mathcal{C}$-oriented line simplification $Q^{\prime}$ with Fréchet distance at most $\epsilon$ to $P$ that has at most as many links as $Q$ has. From Lemma 6.3 follows that there exists a first $q^{\prime} \prec q^{\prime \prime}$ on $Q$ with $N\left(q^{\prime \prime}\right) \prec N\left(q^{\prime}\right)$. Since $Q$ contains no backward extensions it follows that $q^{\prime}$ and $q^{\prime \prime}$ are located in one tube $T_{j}, 1 \leq j \leq n-1$. Let $m$ be a point on $P$ with $N\left(q^{\prime \prime}\right) \prec m \prec N\left(q^{\prime}\right)$. Then $\left\|q^{\prime}-m\right\|>\epsilon$ and $\left\|q^{\prime \prime}-m\right\|>\epsilon$. Let $C_{m}^{\prime}$ be the $\epsilon$-circle around $m$. Particularly, $m$ is a point on link $\overline{p_{j} p_{j+1}}$. There exists a $u \in \partial C_{m}^{\prime} \cap Q$ with $u \preceq w$, for all $w \in \partial C_{m}^{\prime} \cap Q$. Analogously, there exists a $v^{\prime \prime} \in \partial C_{m}^{\prime} \cap Q$ with $w \preceq v^{\prime \prime}$, for all $w \in \partial \bar{C}_{m}^{\prime} \cap Q$. Then, $u$ is a point on $\overline{q_{k-1} q_{k}}$ and $v^{\prime \prime}$ is a point on $\overline{q_{k+l-1} q_{k+l}}$.

Case $l>2$ : Since $v^{\prime \prime}$ can be reached from $u$ inside $C_{m}^{\prime}$ within two $\mathcal{C}$-oriented links, there exists a $w \in C_{m}^{\prime}$ with $\overrightarrow{u w} \in \mathcal{C}$ and $\overrightarrow{w v^{\prime \prime}} \in \mathcal{C}$. Therefore, $Q^{\prime}=\left\langle q_{1}, \ldots, q_{k}, u, w, v^{\prime \prime}, q_{k+l+1}\right.$, $\left.\ldots, q_{m}\right\rangle$ is a $\mathcal{C}$-oriented link path with at most as many links as $Q$ has. For all $q^{\prime} \prec q^{\prime \prime}$ on $\left\langle q_{1}, \ldots, u^{\prime}, w, v^{\prime \prime}, q_{k+l+1}\right\rangle$ we have $N\left(q^{\prime \prime}\right) \nprec N\left(q^{\prime}\right)$.

Case $l=2$ : Then, $\left\|q_{k}-m\right\|>\epsilon$ and $\left\|q_{k+1}-m\right\|>\epsilon$. Let $\overline{q_{k-1} q_{k}} \cap C_{m}^{\prime}=\overline{u v}$ and $\overline{q_{k+1} q_{k+2}} \cap C_{m}^{\prime}=$ $\overline{u^{\prime \prime} v^{\prime \prime}}$. Let $\overline{u v}$ and $\overline{u^{\prime \prime} v^{\prime \prime}}$ have orientation $c$ and $\dot{c}$, respectively. This configuration is very similar to that in Figure 8. We simply have to embed the circle in a tube and rename $p_{e}$ by $m$. Furthermore, analogously to the proof of Lemma 3.6 if follows that there exists a $z \in \overline{u v}$ such that $z+\lambda \bar{c}$ has non empty intersection $z^{\prime}$ with $\overline{u^{\prime \prime} v^{\prime \prime}}$.

Thus, replacing $Q$ by $Q^{\prime}=\left\langle q_{1}, \ldots, q_{k-1}, z, z^{\prime}, q_{k+2}, \ldots, q_{m}\right\rangle$ yields that $Q^{\prime}$ has as many links as $Q$ and for all $q^{\prime} \prec q^{\prime \prime}$ on $\left\langle q_{1}, \ldots q_{k-1}, z, z^{\prime}, q_{k+2}\right\rangle$ we have $N\left(q^{\prime \prime}\right) \nprec N\left(q^{\prime}\right)$.

Remember that we construct the $\mathcal{C}$-oriented line simplification by following the links backwards until we reach the start set $S$. We can construct a $\mathcal{C}$-oriented line simplification with Fréchet distance more that $\epsilon$ to $P$ by the following rule.

Lemma 6.6 Following a link backwards until it hits a point in its generating reachable region for the first time yields an approximation with Fréchet distance at most $\epsilon$ to $P$.

Proof: Assume that the resulting path $Q$ has Fréchet distance more that $\epsilon$ to $P$. Then, we can apply the transformations of the proof of Theorem 6.5 on $Q$. In each transformation step a sequence of links $\left\langle q_{k-1}, \ldots, q_{k+l}\right\rangle$ is replaced by a sequence $\left\langle q_{k-1}, q_{k}^{\prime}, \ldots, q_{k+l-1}^{\prime}, q_{k+l}\right\rangle$, where $q_{k+l-1}^{\prime}$ is a point on $\overline{q_{k+l-1}^{\prime} q_{k+l}}$. Let $q_{k+l-1}$ be reachable with $i$ links. From the transformation

follows that $q_{k+l-1}^{\prime}$ must also be reachable with $i$ links. Since $q_{k+l-1}^{\prime} \prec q_{k+l-1}$ on $\overline{q_{k+l} q_{k+l-1}}$ and the construction of the approximation follows a link backwards until it hits a point in its generating reachable region for the first time we yield a contradiction.

\section{Conclusion}

We presented an algorithm that solves the $\mathcal{C}$-oriented line simplification problem with the additional restriction that the $\mathcal{C}$-oriented polygonal chain has Fréchet distance at most $\epsilon$ to the original chain in polynomial time.

Theorem 7.1 The $\mathcal{C}$-oriented line simplification problem (COLS) with the additional restriction that the $\mathcal{C}$-oriented polygonal chain has Fréchet distance at most $\epsilon$ to the original chain can be solved in $\mathcal{O}\left(k n^{2}|\mathcal{C}|^{2}\left\lceil\frac{\pi}{2 \alpha_{\min }}\right\rceil \log \left(n|\mathcal{C}|\left\lceil\frac{\pi}{2 \alpha_{\min }}\right\rceil\right)\right)$ time. The algorithm needs $\mathcal{O}\left(k n^{2}|\mathcal{C}|^{2}\left\lceil\frac{\pi}{2 \alpha_{\min }}\right\rceil\right)$ space ( $k$ is the minimum number of links, $k \leq 4 n+\sum_{i=1}^{n-1} \frac{\left\|p_{i}-p_{i+1}\right\|_{2}}{2 \epsilon}$ ). 
For sets of orientations $\mathcal{C}$ that do not contain the orthogonal orientation to each orientation we have a negative result:

Corollary 7.2 In case that $\mathcal{C}$ does not contain the orthogonal orientation to each orientation, the minimum $\mathcal{C}$-oriented line simplification can have fewer links than the minimum $\mathcal{C}$-oriented approximation that has Fréchet distance at most $\epsilon$ to $P$.

Proof: Figure 15 shows a polygonal chain and its minimum $\mathcal{C}$-oriented line simplification that has Fréchet distance more than $\epsilon$ to $P$ since $N\left(q^{\prime \prime}\right) \prec N\left(q^{\prime}\right)$ on $P$, with $q^{\prime} \prec q^{\prime \prime}$. Each $\mathcal{C}$-oriented approximation that has Fréchet distance at most $\epsilon$ to $P$ consists of at least 4 links.

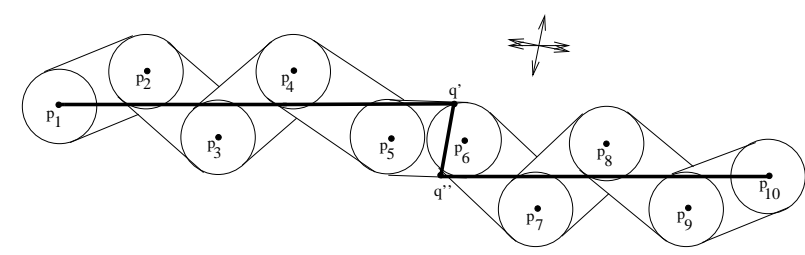

Figure 15: Example for Corollary 7.2.

In case that $\mathcal{C}$ does not contain the orthogonal orientation to each orientation our algorithm still computes a $\mathcal{C}$-oriented approximation but the resulting approximation might neither be minimal, nor have Fréchet distance $\epsilon$. In order to gain minimality it is necessary to allow backward extensions from a tube $T_{j}$ to $T_{k}$ with $k<j$. An open question is how to compute the minimum $\mathcal{C}$-oriented approximation that has Fréchet distance at most $\epsilon$ to $P$ in this case.

The restriction that the $\epsilon$-circles of two consecutive vertices of the original polygonal chain do not intersect is necessary when we want to solve this problem by computing the sets $\mathcal{R} \mathcal{R}^{i}\left(S, T_{j}\right)$ in polynomial time. There exist examples where the number of disjoint reachable regions in $\mathcal{R} \mathcal{R}^{i}\left(S, T_{j}\right)$ gets exponential in $j$ when allowing $C_{l} \cap C_{l+1} \neq \emptyset$. Since the profit of deciphering such an example is rather small we decided not to expose them. The complexity of the $\mathcal{C}$-oriented line simplification problem when allowing $C_{j} \cap C_{j+1} \neq \emptyset$ remains open.

\section{Acknowledgment}

I thank Frank Wagner, Wolfram Schlickenrieder, Arne Storjohann, and Mike Hallett with increasing honor for proof-reading this paper and for valuable comments.

\section{References}

[AG95] Helmut Alt and Michael Godau. Computing the Fréchet distance between two polygonal curves. Internat. J. Comput. Geom. Appl., 5:75-91, 1995.

[AG96] H. Alt and L. J. Guibas. Discrete geometric shapes: Matching, interpolation, and approximation a survey. Research paper B 96-11, Institute of Computer Science, Freie Universität Berlin, 1996.

[AOS94] J. Adegeest, M. Overmars, and J. Snoeyink. Minimum link c-oriented paths: Single-source queries. International Journal of Computational Geometry \&5 Applications, 4(1):39-51, 1994.

[DP73] D. H. Douglas and T. K. Peucker. Algorithms for the reduction of the number of points required to represent a digitized line or its caricature. The Canadian Geographer, 10(2):112122, December 1973.

[GHMS93] L. J. Guibas, J. E. Hershberger, J. S. B. Mitchell, and J. S. Snoeyink. Approximating polygons and subdivisions with minimum link paths. International Journal of Computational Geometry Ef Applications, 3(4):383-415, 1993. 
[God91] M. Godau. Die Fréchet-Metrik für Polygonzüge - Algorithmen zur Abstandsmessung und Approximation. Diplomarbeit, Institute of Computer Science, Freie Universität Berlin, 1991.

[God98] M. Godau. Personal communication. 1998.

[HS92] J. Hershberger and J. Snoeyink. Speeding up the Douglas-Peucker line-simplification algorithm. Technical Report TR-92-07, Department of Computer Science, University of British Columbia, April 1992.

[Wei97] R. Weibel. Generalization of spatial data: Principles and selected algorithms. Lecture Notes in Computer Science, 1340:99-152, 1997. 